\title{
The absorption law
}

\section{Or: how to Kreisel a Hilbert-Bernays-Löb}

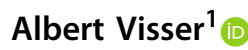

Received: 11 June 2018 / Accepted: 17 November 2020 / Published online: 16 December 2020

(c) The Author(s) 2020

\begin{abstract}
In this paper, we show how to construct for a given consistent theory $U$ a $\Sigma_{1}^{0}$-predicate that both satisfies the Löb Conditions and the Kreisel Condition-even if $U$ is unsound. We do this in such a way that $U$ itself can verify satisfaction of an internal version of the Kreisel Condition.
\end{abstract}

Keywords Provability · Arithmetization · Incompleteness

Mathematics Subject Classification 03F30 - 03F40 - 03F45

\section{Introduction}

When does a predicate $P$ in a theory $U$ count as a provability predicate for $U$ ? There are various ideas on the market to explicate this notion. These ideas provide conditions for being a provability predicate that cater to various intuitions. ${ }^{1}$ In the present paper, three classes of conditions will be considered: the Hilbert-Bernays-Löb Conditions, the Kreisel Condition and the Feferman Condition. We will introduce the various conditions with some care in Sect. 3. In the present paper, we will not go into the philosophical discussion about the meaning of the conditions and their relative pro's and con's. However, in Appendix A, we will give examples that illustrate that all three

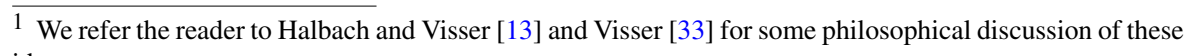
ideas.

I am grateful to Fedor Pakhomov for sharing his ideas about slow provability and for a number of corrections to an earlier version of this paper. I thank Volodya Shavrukov for asking good questions and for pointing me to some references. I am grateful to Michael Beeson for pointing out a mistake and an ambiguity. I thank the anonymous referee for excellent suggestions.

$\bowtie \quad$ Albert Visser

a.visser@uu.nl

1 Faculty of Humanities, Department of Philosophy and Religious Studies, Utrecht University, Janskerkhof 13, 3512BL Utrecht, The Netherlands 
classes of conditions are independent of one another. These examples can help the reader to form her own impression of what the conditions involve and, possibly, help her to get more grip on the issues surrounding the choice between the various classes of conditions.

The aim of our paper is to study the interplay of the Hilbert-Bernays-Löb Conditions and the Kreisel Condition for the case of $\Sigma_{1}^{0}$-predicates. The Kreisel condition for a provability predicate $\triangle$ for a theory $U$ demands that $U \vdash \triangle A$ iff $U \vdash A$. A first question is whether we can have the Kreisel Condition for a predicate that satisfies the Löb Conditions in case our theory $U$ is unsound. For example, what happens when our theory is PA + incon(PA)? A second question is as follows. If $\triangle$ satisfies the Löb Conditions, the theory $U$, when consistent, cannot verify both the Kreisel Condition and the internal Kreisel Condition $\triangle \triangle A \leftrightarrow \triangle A$. However, can we have the next best thing, to wit: given an appropriately good provability predicate $\square$ for $U$, can we find a predicate $\triangle$, that satisfies the Löb Conditions and for which we have both $U \vdash \triangle A$ iff $U \vdash A$, and $U \vdash \square \triangle A \leftrightarrow \square A$ ? As we will see, the answer to the last question is yes. We can find, in many cases, a predicate $\triangle$ that satisfies the Löb Conditions, the Kreisel Condition and the internal Kreisel Condition $U \vdash \square \triangle A \leftrightarrow \square A$.

We develop a general construction of a $\Sigma_{1}^{0}$-predicate $\triangle$ that satisfies both the Löb Conditions and the external and internal Kreisel Conditions from suitable data. The internal Kreisel principle $\vdash \square \triangle A \leftrightarrow \square A$ splits in two sub-principles, to wit, the absorption law $\vdash \square \triangle A \rightarrow \square A$ and the emission law $\vdash \square A \rightarrow \square \triangle A$. Our main focus will be on the absorption law.

\subsection{Historical note}

The following fact is due to Orey. Suppose $U$ is an extension of PA. Then, we can find an elementary $\alpha$ such that $\alpha$ represents the axiom set of $U$ over PA and $U \nvdash \square_{\alpha} \perp$. See [3]. See also [20, Chapter 2].

A construction of a Fefermanian predicate ${ }^{2}$ with $\Sigma_{1}^{0}$-axiomatization $\alpha$ for a theory $U$ that extends PA, such that none of the iterated $\square_{\alpha}$-inconsistency statements $\square_{\alpha}^{n} \perp$ is provable in $U$ is given in [2]. See also [18]. The result we will prove extends the results of Orey and Beklemishev.

The predicates constructed in the present paper can be viewed as slow provability predicates. The absorption law holds for slow provability predicates over PA. Slow provability over EA was introduced and studied in [30]. Slow provability over PA was introduced and studied in [6]. Our knowledge concerning this notion was further extended in $[4,5,11,26]$.

The disadvantage of the present approach to slow provability is that the connection to proof theory and ordinal analysis is not visible. The advantage of the present approach to slow provability compared to the one of Friedman [6] is its wider scope. Moreover, as we discuss below, it is not known whether the approach of Friedman [6] works for Heyting's Arithmetic HA, the constructive counterpart of PA, where

\footnotetext{
2 This means, roughly, that we use a standard arithmetization of syntax and proofs, but tinker just with the representation of the axiom set. See Sect. 3.3, for a detailed explanation.
} 
our approach works without problems. (This does not mean that it would not be very interesting to see how to extend the methods of Friedman [6] to the constructive case.)

An alternative approach to obtain a provability style predicate that satisfies both the Löb Conditions and the Kreisel Condition can be found in Section 5 of [34]. The approach in the present paper has a number of advantages. First, it is somewhat more perspicuous. Secondly, the constructed predicates also satisfy the Hilbert-Bernays Condition. Thirdly, the construction of the predicates is fixed-point-free. Fourthly, using the present approach we can also, in a number of cases, construct predicates $\triangle$ with the desired properties that are Fefermanian (in a sense that will be further specified in the paper).

The basic idea for the predicate constructed in this paper is due to Fedor Pakhomov. He suggested considering this predicate when I asked him whether there was a nonmodel theoretic proof of the absorption law for slow provability. However, the proof of absorption given in this paper is quite different from the one Fedor had in mind.

\subsection{Prerequisites}

The reader should be familiar with basic materials from Hájek and Pudlák [10]. For certain local results there may be further prerequisites, but we will make these clear in situ.

\subsection{Overview of the paper}

In Sect. 2, we introduce the basic facts, notations and definitions for the rest of the paper.

Section 3 is a brief treatment of the Löb Conditions, the Kreisel Condition and the Feferman Condition.

Then, in Sect. 4, we construct a predicate $\triangle$ with the desired properties for theories $U$ that extend Peano Arithmetic. This is, in many respects, the simplest case of the construction. In this simple case, we have the extra property that our $\Delta$ is Fefermanian. On the other hand, the construction is subject to some restrictions. It works only if we start with an elementary numeration $\alpha$ of the axiom set of $U$ such that $\pi \preceq \alpha$. The construction delivers an axiom set numerated by $\widetilde{\alpha}$ for $U$. However, we will have $\pi \npreceq \widetilde{\alpha}$, so that the construction cannot be iterated. The construction in this section will also be covered by the more general construction in Sect. 5. However, we think the general treatment becomes easier to follow if one has seen Sect. 4 first.

In Sect. 5 , we give the basic construction in the abstract, starting from a predicate $\theta$ that satisfies a list of properties. We illustrate how $\square_{\alpha_{x}}$ of Sect. 4 does satisfy the properties for $\theta$, so that the approach of Sect. 4 is subsumed under the approach of Sect. 5.

Finally, in Sect. 6, we show, under fairly general conditions, how to construct a predicate $\theta$ that satifies the desired properties.

In "Appendix A", we provide separation examples between the Löb Conditions, the Kreisel Condition and the Feferman Conditions. 


\section{Basic conventions, notations, definitions}

In this section, we introduce basic conventions and fix some notations and give some definitions.

\subsection{Theories}

A theory $U$ in this paper is a theory in the signature of arithmetic. ${ }^{3}$ A theory is given by a set $X$ of axioms. We will generally assume that $X$ is a recursively enumerable set. However, $X$ is just given as a set and it is not intrinsically connected with a presentation. We will assume as a default that $U$ extends Elementary Arithmetic EA.

Two salient theories of the paper are Elementary Arithmetic EA and Peano Arithmetic PA. The theory EA is I $\Delta_{0}+$ exp. It is finitely axiomatizable by a single axiom $B$. See [10]. The predicate $x=\ulcorner B\urcorner$ will be called $\beta$. The theory PA has a standard elementary presentation $\pi$ of the axiom set corresponding to the usual axiom scheme.

We will also consider the extension of EA with the $\Sigma_{1}^{0}$-collection principle B $\Sigma_{1}^{0}$. This principle is given by:

$$
\vdash \forall x \leq a \exists y S_{0}(x, y) \rightarrow \exists b \forall x \leq a \exists y \leq b S_{0}(x, y) .
$$

Here $S_{0}$ is $\Sigma_{1}^{0}$ and may contain further parameters.

\subsection{Arithmetization}

We will sometimes use implementation properties of the arithmetization like monotonicity and the efficiency of syntactical operations. For this reason, we outline a few features of the Gödel coding we intend to use. We use a style of Gödel numbering that is due to Smullyan (see [27]). Our Gödel numbering is based on the length-first ordering. We enumerate the strings of our finite alphabet according to length and the strings of the same length alphabetically. The Gödel number of a string $s$ will be the number of occurrences in this enumeration. In this ordering the arithmetical function tracing concatenation is of the order of multiplication. We can use our bijective coding of strings to implement sequences of numbers. This has the bonus that also concatenation of sequences of numbers will be of the order of multiplication. ${ }^{4}$

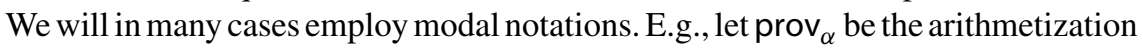
of provability from the axioms in $\alpha$. We write $\square_{\alpha} A$ for $\operatorname{prov}_{\alpha}(\ulcorner A\urcorner)$. Here $\ulcorner A\urcorner$ is the numeral of the Gödel number of $A$. We will sometimes quantify the sentence-variables

\footnotetext{
${ }^{3}$ Everything in the paper lifts to the more general case where a theory of arithmetic is interpretable in the given theory. However, it is pleasant to avoid the extra notational burden of the more general case. The notational burdens of the present paper seem to be sufficiently heavy already.

4 Usually, there is some overhead in defining sequences since we want to add some materials to make the definition of the projection function easy. However, the uses of sequences to define syntax and proofs usually only require that we can determine whether something occurs in a sequence before something else. For this one does not need the extra material.
} 
inside a modal operator. For example, we write things like:

$$
(\dagger) \forall A, B\left(\left(\square_{\alpha} A \wedge \square_{\alpha}(A \rightarrow B)\right) \rightarrow \square_{\alpha} B\right) \text {. }
$$

This stands for:

$$
\text { (†) } \forall x \forall y \forall z\left(\left(\operatorname{prov}_{\alpha}(x) \wedge \operatorname{imp}(x, y, z) \wedge \operatorname{prov}_{\alpha}(z)\right) \rightarrow \operatorname{prov}_{\alpha}(y)\right)
$$

Admittedly, such notations are somewhat sloppy, but I think in practice they are very convenient. E.g., $(\dagger)$ is more pleasant to read than ( $\ddagger)$.

We employ the usual conventions for quantifying numerical variables into modal contexts. E.g. $\square_{\alpha} A(x)$ means $\exists z\left(\operatorname{sub}\left(x,\left\ulcorner v_{0}\right\urcorner,\left\ulcorner A\left(v_{0}\right)\right\urcorner, z\right) \wedge \operatorname{prov}_{\alpha}(z)\right)$.

We will employ the witness comparison notation. Suppose $A=\exists x A_{0}(x)$ and $B=\exists x B_{0}(x)$. We write:

- $A \leq B$ for $\exists x\left(A_{0}(x) \wedge \forall y<x \neg B_{0}(y)\right)$.

- $A<B$ for $\exists x\left(A_{0}(x) \wedge \forall y \leq x \neg B_{0}(y)\right)$.

\subsection{Ordering of predicates for axioms}

Let $\gamma(x)$ and $\delta(x)$ be formulas with only $x$ free that EA-verifiably represent classes of arithmetical sentences. Let $T$ be an extension of EA. We write $\gamma \preceq_{T} \delta$ for

$$
T \vdash \forall A\left(\operatorname{prov}_{\gamma}(A) \rightarrow \operatorname{prov}_{\delta}(A)\right)
$$

Here $\operatorname{prov}_{\alpha}$ is a standard arithmetization of provability from $\alpha$. Our default for $T$ will be EA and we will write $\preceq$ for $\preceq$ EA.

It is easy to see that $\preceq_{T}$ is a partial pre-ordering.

\section{Conditions for provability predicates}

In this section, we introduce three (classes of) conditions that aim to explicate when a predicate is a provability predicate.

\subsection{The Löb conditions}

To state the Löb conditions we write $\triangle A$ for $P(\ulcorner A\urcorner)$ and $\vdash$ for provability in $U$. The Löb conditions (introduced in [21]) are as follows.

L1. If $\vdash A$, then $\vdash \triangle A$

L2. $\vdash(\triangle A \wedge \triangle(A \rightarrow B)) \rightarrow \triangle B$

L3. $\vdash \triangle A \rightarrow \triangle \triangle A$

We obtain the Hilbert-Bernays Conditions in case we replace L3 by:

HB. $\vdash S \rightarrow \triangle S$, for $\Sigma_{1}^{0}$-sentences $S$. 
The usual assumption connected to the Hilbert-Bernays conditions is that $P$ be $\Sigma_{1}^{0}$, so that $\mathrm{L} 3$ is a special case of HB. It is easy to see that if $P$ is not $\Sigma_{1}^{0}$, we can have L1, 2 and HB but not L3. E.g., we may take $P$ to be Feferman provability for PA.

We note that, in case $P$ is $\exists \Sigma_{1}^{\mathrm{b}}$, the Löb conditions are more general than the Hilbert-Bernays Conditions. For example, in a weak theory like $S_{2}^{1}$ we do have the Löb Conditions for $S_{2}^{1}$-provability for a standard provability predicate-assuming an efficient arithmetization-but it is unknown whether we have the Hilbert-Bernays Conditions.

Technically, the Löb Conditions constitute a superior analysis of the proof of the Second Incompleteness Theorem. The philosophical use of the Conditions is independent of their technical interest. The philosophical idea is that the Löb Conditions explicate the theoretical role that a provability predicate plays in a theory.

We note that the Löb conditions do depend on the choice of Gödel numbering and hence are still not entirely 'coordinatefree'. For a study of this dependence and a proposal to abstract away from it, see [7].

The Löb Conditions also have a uniform and a global version. In the uniform version we allow parameters in the formulas inside the operator. For example, L2 becomes: $\vdash \forall \vec{x}((\triangle A(\vec{x}) \wedge \triangle(A(\vec{x}) \rightarrow B(\vec{x}))) \rightarrow \triangle B(\vec{x}))$. In the global version, the quantifiers over sentences are not outside but inside the theory. For example, L2 becomes: $\vdash$ $\forall A, B \in$ sent $((\triangle A \wedge \Delta(A \rightarrow B)) \rightarrow \triangle B)$. We note that the global version is stronger than the uniform one. We will not consider the strengthened conditions in the present paper.

\subsection{The Kreisel condition}

The Kreisel Condition was first formulated in [16]. Its statement is as follows:

K. $U \vdash \triangle A$ iff $U \vdash A$.

We note that the Kreisel Condition is of a quite different nature than the Löb Conditions. It just asks that the theory numerates its own provability by the given predicate.

One could imagine a variant of the Kreisel Condition where we just ask numerability in a base theory $U_{0}$ that is a sub-theory of $U$.

Finally, we observe that, like the Löb Conditions, the Kreisel Condition does depend on the chosen Gödel numbering.

\subsection{The Feferman condition}

We explain the idea that a provability-predicate is Fefermanian. We derive this idea from the methodology introduced in [3]. The main ingredient of the idea is simply to fix a preferred arithmetization of provability and allow the choice of the predicate $\alpha$ representing the axiom-set to be free, given that it satisfies certain adequacy conditions.

The best way to present a Fefermanian predicate is to view it as a tuple $\left\langle U_{0}, U, \alpha\right\rangle$. Here $U_{0}$ is the base theory and $U$ is the lead theory. We ask that $U$ extends the base $U_{0}$. We demand that $\alpha$ numerates an axiom set $X$ for $U$ in the base theory $U_{0}$. In other words, we demand that $A \in X$ iff $U_{0} \vdash \alpha(\ulcorner A\urcorner)$. 
We note that the demands on a Fefermanian predicate treat the axioms of the lead theory via a condition similar to the Kreisel Condition.

In the present paper, we will consider Fefermanian predicate modulo provability in the base theory. Thus, we will say that $P$ is Fefermanian for $U$ over $U_{0}$ in the relaxed sense iff, there is an $\alpha$ such that $\left\langle U_{0}, U, \alpha\right\rangle$ is Fefermanian in the strict sense and $U_{0} \vdash \forall x\left(P(x) \leftrightarrow \operatorname{prov}_{\alpha}(x)\right)$.

The reader may object that the Feferman Condition does not count as a real condition since it employs an unspecified specification of the arithmetization. ${ }^{5}$ Of course, the reader is correct here. Feferman, in his paper, does specify a choice for a proof system and an arithmetization. However, in Feferman's arithmetization, the Gödel number of a formula is superexponential in its length, so it is not a convenient Gödel numbering to work with within EA. Moreover, if Feferman's specific Gödel numbering would really be the golden standard, it would be reasonable that everybody would know its specification, but, of course, that is not the case. I see the use of the Feferman idea more as dialogical. The reader is asked to take her favored good arithmetization in mind and read for prov provability according to that arithmetization. So, prov becomes context dependent like the word 'you'. I will employ the Feferman idea in this way.

\subsection{Properties of Fefermanian predicates}

In this subsection we briefly consider some basic insights on Fefermanian predicates.

Let $\mathrm{A}_{U}$ be the class of all $\alpha$ in $\Sigma_{1}^{0}$ such that $\langle\mathrm{EA}, U, \alpha\rangle$ is Fefermanian.

Theorem 1 Let $U$ be a theory. Then $\mathrm{A}_{U}$ has a minimum w.r.t. $\preceq$ iff $U$ is finitely axiomatizable.

Proof Suppose $U$ is finitely axiomatizable, say by $A_{0}, \ldots, A_{n-1}$. Let $\alpha^{*}(x):=$ $\bigvee_{i<n} x=\left\ulcorner A_{i}\right\urcorner$. Consider any $\alpha$ in $\mathrm{A}_{U}$. We find for $i<n$ that $U \vdash A_{i}$, and, hence $\mathrm{EA} \vdash \bar{\square}_{\alpha} A_{i}$.

We reason in EA. Suppose $p$ witnesses $\square_{\alpha^{*}} A$ and $p_{i}$, for $i<n$, witnesses $\square_{\alpha} A_{i}$. We obtain an $\alpha$-proof $q$ of $A$ by adding the $p_{i}$ 'above' $A_{i}$ to $p$. (Note that we do not need $\Sigma_{1}^{0}$-collection since $n$ is standard.)

Suppose $U$ is not finitely axiomatizable. Consider any $\alpha \in \mathrm{A}_{U}$. Clearly, for any $n$ there is a $B$ such that $U \vdash B$ but the axioms in $\alpha$ that are $\leq n$ do not prove $B$. Hence, $C:=\forall x \exists B\left(\square_{\alpha} B \wedge \neg \square_{\alpha_{x}} B\right)$ is true, where $\alpha_{x}(y): \leftrightarrow \alpha(y) \wedge y \leq x$. Thus, EA $+C$ is consistent. Let $\gamma(x): \leftrightarrow \beta(x) \vee x=\ulcorner C\urcorner$, where $\beta$ is the standard axiomatization of EA. We define:

$$
\alpha^{\prime}(x): \leftrightarrow \alpha(x) \wedge \forall y \leq x \neg \operatorname{proof}_{\gamma}(x,\ulcorner\perp\urcorner) .
$$

It is evident that $\alpha^{\prime} \preceq \alpha$. Suppose $(\dagger) \alpha \preceq \alpha^{\prime}$. We reason inside EA $+C$. By $(\dagger)$, we have $\forall B\left(\square_{\alpha} B \rightarrow \square_{\alpha^{\prime}} B\right)$. Suppose $p$ is a $\gamma$-proof of $\perp$. It follows that the $\alpha^{\prime}$ axioms are below $p$. Consider $B$ such that $\square_{\alpha} B$ but not $\square_{\alpha_{p}} B$. It follows that $\neg \square_{\alpha^{\prime}} B$. A contradiction. It follows that there is no $\gamma$-proof of $\perp$, in other words, $\diamond_{\gamma} \top$. We leave $\mathrm{EA}+C$.

\footnotetext{
5 As remarked above the other conditions suffer, admittedly to a lesser degree, from the same defect.
} 
We have shown EA $+C \vdash \diamond_{\gamma} \top$. But this contradicts the Second Incompleteness Theorem. Hence $(\dagger)$ must fail.

Remark 1 What happens if we replace EA in the definition of $A_{U}$ by another base theory $T$ and, simultaneously, consider $\preceq_{T}$ in stead of $\preceq_{\mathrm{EA}}$ ? Inspection of the proof of Theorem 1 shows that we have to replace $\beta$ in the proof by an elementary predicate numerating the axioms of $T$ in $T$. This can always be arranged due to Craig's trick. We also have to assume that $T$ is $\Sigma_{2}^{0}$-sound to be sure that $T+C$ is consistent. We note that the application of the Second Incompleteness theorem goes through by the usual argument since the analogue of $\gamma$ is elementary. ${ }^{6}$

Thus, our result goes through, as long as the base theory is $\Sigma_{2}^{0}$-sound.

Theorem 2 Consider theories $U_{0}$ and $U$ where EA is a sub-theory of $U_{0}$ and $U_{0}$ is a sub-theory of $U$. Suppose:
a. $P$ numerates $U$ in $U_{0}$.
b. $P$ contains $U_{0}$-provably all predicate-logical tautologies.
c. $P$ is $U_{0}$-provably closed under finite conjunctions.
d. $P$ is $U_{0}$-provably closed under modus ponens.

Then, $P$ is Fefermanian (in the relaxed sense) for $U$ over $U_{0}$ as witnessed by $\left\langle U, U_{0}, P\right\rangle$.

Proof Clearly, we have $U_{0} \vdash \forall B \in P \operatorname{prov}_{P}(B)$. Conversely, reason in $U_{0}$. Suppose $p$ is a $P$-proof of $B$. Let $X$ be the finite set of $P$-axioms used in $p$. Then, $(\bigwedge X \rightarrow B)$ is a predicate logical tautology, so $(\bigwedge X \rightarrow B) \in P$. By closure under conjunction, we have $\bigwedge X \in P$. Hence, by closure under modus ponens, we find $B \in P$.

Theorem 3 Consider theories $U_{0}$ and $U$ where $\mathrm{I} \Sigma_{1}^{0}$ is a sub-theory of $U_{0}$ and $U_{0}$ is a sub-theory of $U$. Let $P$ be a $\Sigma_{1}^{0}$-predicate. Suppose:
a. $P$ numerates $U$ in $U_{0}$.
b. $P$ contains $U_{0}$-provably all predicate-logical tautologies.
c. $P$ is $U_{0}$-provably closed under modus ponens.

Then $P$ is Fefermanian for $U$ over $U_{0}$ with $P$ itself as representation of the axiom set.

Proof Under the assumptions of the theorem, we can prove that $P$ is closed under finite conjunctions by $\Sigma_{1}^{0}$-induction.

Example 1 We take as base and lead theory PA. The predicate $\square_{\pi} \square_{\pi}$ is Fefermanian. Similarly, for $\exists x \square_{\pi}^{x+1}(\cdot)$. The last predicate is, modulo PA-provable equivalence, Parikh provability or fast provability. Parikh provability can be obtained by adding to an axiomatization based on $\pi$ the Reflection Rule: $\vdash \square_{\pi} A \Rightarrow \vdash A$. See [24]. See also [9].

Theorem 4 Suppose $U$ extends EA and $P$ is Fefermanian w.r.t. a $\Delta_{0}(\exp )$-presentation $\alpha$ of the axiom set. Then, $P$ satisfies the Löb Conditions.

\footnotetext{
6 If we consider a $\Sigma_{1}^{0}$-axiomatization in a context without $\Sigma_{1}^{0}$-collection, the Löb conditions may fail. However, even in such Löbless cases, the Second Incompleteness Theorem holds. See [35].
} 
Theorem 5 Suppose $U$ extends $\mathrm{EA}+\mathrm{B} \Sigma_{1}^{0}$ and $P$ is Fefermanian w.r.t. a $\Sigma_{1}$ presentation $\alpha$ of the axiom set. Then, $P$ satisfies the Löb Conditions.

Theorem 6 Suppose $\left\langle U_{0}, U, \alpha\right\rangle$ is a strict Fefermanian representation, where $\alpha$ is $\Sigma_{1}^{0}$, and suppose $U$ and $U_{0}$ are sound. Then, $\square_{\alpha}$ satisfies the Kreisel Condition for $U$.

Proof Since $U_{0}$ is sound, we have $\alpha(\ulcorner A\urcorner)$ iff $U_{0} \vdash \alpha(\ulcorner A\urcorner)$. So, $\alpha$ truly represents the axioms of $U$. It follows that $U \vdash A$ iff $\square_{\alpha} A$. Since $\bar{U}$ is sound, we find $\square_{\alpha} A$ iff $U \vdash \square_{\alpha} A$. So, we may conclude $U \vdash A$ iff $U \vdash \square_{\alpha} A$.

Finally, we look into the interaction of elementary axiomatizations, i.e., $\Delta_{0}$ (exp)axiomatizations, and $\Sigma_{1}^{0}$-axiomatizations. We note that an elementary formula numerates the same set in every consistent theory. This immediately gives us the following insight.

Theorem 7 Suppose $U_{0}$ and $U_{1}$ are consistent subtheories of $U$. Suppose further that $\left\langle U_{0}, U, \alpha\right\rangle$ is Fefermanian, where $\alpha$ is elementary. Then, $\left\langle U_{1}, U, \alpha\right\rangle$ is Fefermanian.

Similarly, a $\Sigma_{1}^{0}$-formula numerates the same set in all $\Sigma_{1}^{0}$-sound theories. So, we have:

Theorem 8 Suppose $U_{0}$ and $U_{1}$ are $\Sigma_{1}^{0}$-sound subtheories of $U$. Suppose further that $\left\langle U_{0}, U, \alpha\right\rangle$ is Fefermanian, where $\alpha$ is $\Sigma_{1}^{0}$. Then, $\left\langle U_{1}, U, \alpha\right\rangle$ is Fefermanian.

We remind the reader of Craig's trick. Let $U_{0}$ be a $\Sigma_{1}^{0}$-sound base theory. Suppose $\sigma$ is a $\Sigma_{1}^{0}$-formula that $U_{0}$-provably represents a set of arithmetical sentences. Suppose $\sigma(x)=\exists y \sigma_{0}(y, x)$, where $\sigma_{0} \in \Delta_{0}(\exp )$. We define

$$
\hat{\sigma}(x): \leftrightarrow \exists y \leq x \exists z \leq x\left(\sigma_{0}(y, z) \wedge x=\operatorname{conj}(\operatorname{id}(\operatorname{num}(y), \operatorname{num}(y), z))\right) .
$$

Here conj arithmetizes forming a conjunction, id arithmetizes forming an identity statement from terms, num arithmetizes the numeral function. Clearly, $\hat{\sigma}$ is elementary. As is well known, we have:

Theorem 9 i. EA $\vdash A\left(\square_{\hat{\sigma}} A \rightarrow \square_{\sigma} A\right)$.

ii. $\mathrm{EA}+\mathrm{B} \Sigma_{1} \vdash \forall A\left(\square_{\hat{\sigma}} A \leftrightarrow \square_{\sigma} A\right)$.

Theorem 10 Suppose $\left\langle U_{0}, U, \sigma\right\rangle$ is Fefermanian and $U_{0}$ is $\Sigma_{1}^{0}$-sound and $\sigma$ is $\Sigma_{1}^{0}$. Then, $\left\langle U_{0}, U, \hat{\sigma}\right\rangle$ is also Fefermanian.

Proof Let $X$ be the set of axioms numerated by $\sigma$ in $U_{0}$ and let $\hat{X}$ be $\hat{\sigma}$ in $U_{0}$. By the $\Sigma_{1}^{0}$-soundness of $U_{0}$, the set $X$ is the set of numbers for which $\sigma$ is true and the set $\hat{X}$ is the set of numbers for which $\hat{\sigma}$ is true. So, by the unformalized version of Theorem 9(ii), we find that both $X$ and $\hat{X}$ axiomatize the same theory, to wit $U$.

We note that it is essential that $U_{0}$ is $\Sigma_{1}^{0}$-sound. If it were not the Craig construction could transform a standard axiom to a non-standard axiom. The non-standard axiom would not be visible in the numeration. 


\subsection{Examples}

We provide a list of examples for coincidence and separation of the conditions. As before $\beta$ is the standard representation of the axiom of EA and $\pi$ is the standard representation of the axioms set of Peano Arithmetic. We will, in our examples, prefer EA over PA, $\Sigma_{1}^{0}$-predicates over more complex ones, and sound theories over unsound ones. Only in the first examples of Examples 8 and 10, perhaps, improvement is possible by finding an example that works for and over EA.

\begin{tabular}{lllllll}
\hline & Base & Lead & $P$ & Löb & Kreisel & Feferman \\
\hline Example 4 & EA & EA & $\Sigma_{1}^{0}$ & + & + & + \\
Example 5 & EA & EA & $\Sigma_{1}^{0}$ & + & + & - \\
Example 6 & EA & EA $+\square_{\beta} \perp$ & $\Sigma_{1}^{0}$ & + & - & + \\
& EA & EA & $\Sigma_{2}^{0}$ & & & \\
Example 7 & EA & EA & $\Sigma_{1}^{0}$ & + & - & - \\
Example 8 & PA & PA & $\Sigma_{2}^{0}$ & - & + & + \\
& EA & EA & $\Sigma_{1,1}^{0}$ & & & \\
Example 9 & EA & EA & $\Sigma_{1}^{0}$ & - & + & - \\
Example 10 & PA & PA & $\Sigma_{2}^{0}$ & - & - & + \\
& EA & EA $+\square_{\beta} \square$ 足 & $\Sigma_{1,1}^{0}$ & & & \\
Example 11 & EA & EA & $\Sigma_{1}^{0}$ & - & - & - \\
\hline
\end{tabular}

We will provide and verify the promised examples in "Appendix A".

\section{Extensions of Peano arithmetic}

Let $U$ be a consistent extension of PA and let $\alpha$ be an elementary numeration of an axiom set $X$ of $U$ in $U$, such that $\pi \preceq \alpha$. ${ }^{7}$ We will show how to construct a $\Sigma_{1}^{0}$ predicate $\widetilde{\alpha}$ that numerates the the axioms of $U$ in $U$. Thus $\widetilde{\alpha}$ will be Fefermanian for $U$ over $U$.

The concrete examples to keep in mind are the standard representation $\pi$ of the axioms of PA and $\pi(x) \vee x=\left\ulcorner\square_{\pi} \perp\right\urcorner$ representing the axioms of PA $+\square_{\pi} \perp$.

We write $\alpha_{x}$ for the $\alpha$-axioms $\leq x$. So, $\alpha_{x}(y): \leftrightarrow \alpha(y) \wedge y \leq x$. We write $\square_{\alpha,(x)}$ for provability from $\alpha$ by a proof $\leq x .{ }^{8}$ We will use $S, S^{\prime}, \ldots$ as variables ranging over $\Sigma_{1}^{0}$-sentences.

A number $x$ is small, or $\mathcal{S}(x)$, iff $\square_{\alpha,(x)}$ is $\Sigma_{1}^{0}$-reflecting. The means that $\mathcal{S}(x)$ iff $\forall S\left(\square_{\alpha,(x)} S \rightarrow \operatorname{true}(S)\right)$, where true is a standard $\Sigma_{1}^{0}$-truth predicate. We note that smallness does depend on the chosen $\alpha$. We also note that, by our assumptions on the Gödel numbering, the quantifier over $S$ can be bounded by $n$. It follows, by $\Sigma_{1}^{0}$ -

\footnotetext{
${ }^{7}$ Our argument also works under the weaker assumption that $\pi \preceq \mathrm{PA} \alpha$.

${ }^{8}$ I use the round brackets to distinguish the intended notion from $\square_{\alpha, x}$ which is used in some of the literature for $\square_{\alpha_{x}}$, where $\alpha_{x}(y): \leftrightarrow \alpha(x) \wedge y \leq x$. 
collection, that, modulo PA-provability, smallness is a $\Sigma_{1}^{0}$-predicate. Finally, smallness is clearly downward closed.

It is consistent with $U$ that not all numbers are small, since $U$ does not prove $\Sigma_{1}^{0}$ reflection for $\alpha$-provability. On the other hand, for every $n$, we have that $U$ proves that it is small, i.e., $U \vdash \mathcal{S}(\underline{n})$. The argument looks like this. (A more general argument is given in the proof of Lemma 2.) Consider a number $n$.

Let $k \leq n$ and $s \leq n$. If $s$ is a code of a $\Sigma_{1}^{0}$-sentence $S$ and if $k$ is the Gödel number of an $\alpha$-proof of $S$, then we have $U \vdash S$, and, hence,

$$
U \vdash\left(\operatorname{sent}_{\Sigma_{1}^{0}}(s) \wedge \operatorname{proof}_{\alpha}(k, s)\right) \rightarrow \operatorname{true}(s) .
$$

If $s$ is not a code of a $\Sigma_{1}^{0}$-sentence or, if $s$ is a code of a $\Sigma_{1}^{0}$-sentence $S$ and $k$ is not the Gödel number of an $\alpha$-proof of $S$, then we have $U \vdash \neg \operatorname{sent}_{\Sigma_{1}^{0}}(s) \vee \neg \operatorname{proof}_{\alpha}(\underline{k}, s)$, and, hence, again, $U \vdash\left(\operatorname{sent}_{\Sigma_{1}^{0}}(s) \wedge \operatorname{proof}_{\alpha}(\underline{k}, s)\right) \rightarrow \operatorname{true}(s)$. It follows that:

$$
U \vdash \bigwedge_{k \leq n, s \leq n}\left(\left(\operatorname{sent}_{\Sigma_{1}^{0}}(s) \wedge \operatorname{proof}_{\alpha}(\underline{k}, s)\right) \rightarrow \operatorname{true}(s)\right) .
$$

Hence, by $U$-reasoning, $U \vdash \mathcal{S}(\underline{n})$. The above reasoning is so simple that it can be verified in PA, and so ( $\dagger)$ PA $\vdash \forall x \square_{\alpha} \mathcal{S}(x)$. The principle ( $\dagger$ ) is a typical example of an outside-big-inside-small principle. Objects that are very big in the outer world are small in the inner world.

We define the slow provability of $A$ or $\triangle A$ as: $A$ is provable from small $\alpha$-axioms. So,

- $\widetilde{\alpha}(x): \leftrightarrow \alpha(x) \wedge \mathcal{S}(x)$

- $\triangle A: \leftrightarrow \square \widetilde{\alpha} A$.

We list two formulas that are equivalent to $\triangle A$ over PA and all provide worthy ways of looking at it. Let $\square_{\alpha}^{*} A: \leftrightarrow \exists x \square_{\alpha_{x}} A$.

- $\triangle A$ iff $\exists x\left(\square_{\alpha_{x}} A \wedge \mathcal{S}(x)\right)$,

- $\triangle A$ iff $\square^{*} A<\exists x \neg \mathcal{S}(x)$.

Remark 2 Our $\triangle$ is part of a family of closely related predicates. To make this visible we consider a slight variant of our $\Delta$ with the same good properties. We write $\square_{\alpha}^{\Pi_{1}^{0}}$ for provability with a $\Pi_{1}^{0}$-oracle. We define $\triangle^{\circ} A$ as $\square_{\alpha}^{*} A<\square_{\alpha}^{\Pi_{1}^{0}} \perp$. We note that this is equivalent to $\exists x\left(\square_{\alpha_{x}} A \wedge \diamond_{\alpha,(x)}^{\Pi_{1}^{0}} \top\right)$.

This representation brings out the analogy with Feferman provability which can be defined as $\square_{\alpha}^{*} A<\square_{\alpha}^{*} \perp$ and a provability predicate studied in [12,14], to wit $\exists x\left(\square_{\alpha_{x+1}} A \wedge \diamond_{\alpha_{x}}^{\Pi_{1}^{0}} \top\right)$ or, alternatively, $\square_{\alpha}^{*} A \leq \square_{\alpha}^{* \Pi_{1}^{0}} \perp$. We note that, unlike $\triangle^{\circ}$, these predicates are not $\Sigma_{1}^{0}$.

Suppose $A$ is in $X$. Then, $U \vdash \alpha(\ulcorner A\urcorner)$. Since also $U \vdash \mathcal{S}(\ulcorner A\urcorner)$, we find $U \vdash$ $\widetilde{\alpha}(\ulcorner A\urcorner)$. Conversely, suppose $U \vdash \tilde{\alpha}(\ulcorner A\urcorner)$. Then, $U \vdash \alpha(\ulcorner A\urcorner)$ and, hence, $A \in X$. Thus $\widetilde{\alpha}$ numerates $X$ in $U$. 
Since $\triangle$ is $\square_{\widetilde{\alpha}}$ and $\widetilde{\alpha}$ is $\Sigma_{1}^{0}$, it follows, in PA by $\Sigma_{1}^{0}$-collection, that that $\triangle$ is $\Sigma_{1}^{0}$. Hence, $\triangle$ satisfies the Löb conditions.

We show that PA verifies emission and absorption for $\triangle$. By the soundness of PA, the Kreisel Condition follows.

We first prove emission. We prove the stronger PA $\vdash \square_{\alpha} A \rightarrow \square_{\alpha} \triangle A$. We reason in PA. Suppose $\square_{\alpha} A$. Then, clearly, for some $x$, we have $\square_{\alpha_{x}} A$. Hence, $\square_{\alpha} \square_{\alpha_{x}} A$. Also, ( $\dagger)$ gives us $\square_{\alpha} \mathcal{S}(x)$. So, $\square_{\alpha}\left(\square_{\alpha_{x}} A \wedge \mathcal{S}(x)\right)$ and, thus, $\square_{\alpha} \triangle A$.

We prove absorption. The proof turns out to be remarkably simple. We find $R$ such that $\mathrm{EA} \vdash R \leftrightarrow\left(\exists x \square_{\alpha_{x}} A\right)<\square_{\alpha} R$. We note that $R$ is $\Sigma_{1}^{0}$.

We reason in PA. Suppose $\square_{\alpha} \triangle A$. We prove $\square_{\alpha} A$. We reason inside $\square_{\alpha}$. Since, by assumption, $\triangle A$, we have, for some $x$, (i) $\square_{\alpha_{x}} A$ and (ii) $\forall S$ ( $\square_{\alpha,(x)} S \rightarrow \operatorname{true}(S)$ ). In case not $\square_{\alpha,(x)} R$, by (i), we find $R$. If we do have $\square_{\alpha,(x)} R$, we find $R$ by (ii). We leave the $\square_{\alpha}$-environment. We have shown $\square_{\alpha} R$. It follows, (a) that for some $p$, we have $\square_{\alpha} \square_{\alpha,(p)} R$ and, by the fixed point equation for $R$, (b) $\square_{\alpha}\left(\left(\exists x \square_{\alpha_{x}} A\right)<\square_{\alpha} R\right)$. Combining (a) and (b), we find $\square_{\alpha} \square_{\alpha_{p}} A$, and, thus, since $U$, as axiomatized by $\alpha$, is, EA-verifiably, essentially reflexive by our assumption that $\pi \preceq \alpha$, we obtain $\square_{\alpha} A$, as desired. We leave PA. We have shown PA $\vdash \square_{\alpha} \triangle A \rightarrow \square_{\alpha} A$.

What happens if we drop the assumption that $\alpha$ is elementary and work with a $\Sigma_{1}^{0}$-predicate $\sigma$ ? We have the following.

Theorem 11 Suppose $\langle\mathrm{PA}, U, \sigma\rangle$ is Fefermanian, where $\sigma$ is $\Sigma_{1}^{0}$ and $\pi \preceq \sigma$. Then we can construct a $\Sigma_{1}^{0}$-predicate $\sigma^{*}$ such that $\left\langle U, U, \sigma^{*}\right\rangle$ is Fefermanian and $\square_{\sigma^{*}}$ satisfies, in $U$, the Löb-conditions, the Kreisel condition and the pair $\square_{\sigma^{*}}, \square_{\sigma}$ satisfies emission and absorption over $U$.

Proof We take $\sigma^{*}$ to be $\tilde{\hat{\sigma}}$. We note that $\langle\mathrm{PA}, U, \hat{\sigma}\rangle$ is Fefermanian and thus $\square_{\sigma^{*}}$ satisfies, in $U$, the Löb-conditions and the Kreisel condition. Moreover the pair $\square_{\sigma^{*}}$, $\square_{\hat{\sigma}}$ satisfies emission and absorption over $U$. However, $\square_{\sigma}$ and $\square_{\hat{\sigma}}$ are co-extensional over PA and, hence, a fortiori, over $U$. So, the pair $\square_{\sigma^{*}}, \square_{\sigma}$ also satisfies emission and absorption over $U$.

Remark 3 The arguments of this section can be extended to constructive logic. In this case we still have the representations $\beta$ for the axiom set of i-EA and $\pi$ for the axiom set of HA. So the whole development remains unchanged. One just has to check that never a step was taken that is essentially classical.

The intuitionistic development has an important point. In their paper [1], Mohammad Ardeshir and Mojtaba Mojtahedi characterize the provability logic of HA for $\Sigma_{1}^{0}$-substitutions. This is the most informative result on the provability logic of HA at the moment of writing. An alternative proof has been developed in [36]. This proof uses slow provability in the style of Friedman, Rathjen and Weiermann for HA. The proof works because only a restricted version of the absorption law is needed. The validity of the full absorption law is plausible but not proved. Replacement by of Friedman-Rathjen-Weiermann slow provability by slow provability in the style of the present paper (as suggested by Fedor Pakhomov) does give us full absorption.

We show that we get a strengthened version of absorption in the case of HA. The proof is intended for readers with some background in the metamathematics of constructive arithmetical theories. 
Theorem 12 i-EA $\vdash \square_{\pi}(A \vee B) \leftrightarrow \square_{\pi}(A \vee \square \widetilde{\pi} B)$.

Proof We reason in i-EA.

Suppose $\square_{\pi}(A \vee B)$. It follows by either q-realizability or the de Jongh translation that, for some $x$, we have $\square_{\pi}\left(A \vee \square_{\pi_{x}} B\right)$. From this, we may conclude $\square_{\pi}(A \vee \square \widetilde{\pi} B)$.

Conversely, suppose $\square_{\pi}\left(A \vee \square_{\tilde{\pi}} B\right)$. By the left-to-right case (with change of variables), we have $\square_{\pi}\left(\square_{\tilde{\pi}} A \vee \square_{\tilde{\pi}} B\right)$. Hence, $\square_{\pi} \square \widetilde{\pi}(A \vee B)$. So, by absorption, $\square_{\pi}(A \vee B)$.

Thus, the alternative predicates that satisfy the absorption law also have a rich constructive life.

\section{The abstract construction}

In this section we present a construction that builds an appropriate $\Delta$ from a given predicate $\theta$ that satisfies certain good properties.

As before the variables $S, S^{\prime}, \ldots$ will range over (codes of) $\Sigma_{1}^{0}$-sentences.

\subsection{The argument}

Let $U$ be a theory. Suppose $\alpha(x)$ is an elementary predicate that numerates the axioms of $U$ in $U$. Let $\theta(y, z)$ be a $\Sigma_{1}^{0}$ binary predicate. We demand that $\theta$ is EA-verifiably, upwards persistent in $y$, i.e., we assume that

$$
\mathrm{EA} \vdash\left(\theta(y, z) \wedge y<y^{\prime}\right) \rightarrow \theta\left(y^{\prime}, z\right) .
$$

Let $\square_{\theta, y} A$ be $\theta(y,\ulcorner A\urcorner)$. We write $\square_{y} A$ as long as $\theta$ is given in the context.

As a heuristic, the reader may think of $\square_{\theta, y} A$ as a generalization of $\square_{\alpha_{y}} A$ as we used it in Sect. 4, studying the case where $\pi \preceq \alpha$.

We define:

- true is the $\Sigma_{1}^{0}$-truth predicate, which is of the form $\exists y \operatorname{true}_{0}(y, x)$, where true $e_{0}$ is $\Delta_{0}(\exp )$. We write $\operatorname{true}^{z}(x)$ for $\exists y \leq z \operatorname{true}_{0}(y, x)$.

- $\square_{\alpha,(x)} A: \leftrightarrow \exists p \leq x$ proof $_{\alpha}(p,\ulcorner A\urcorner)$, where proof is the standard arithmetization of the proof predicate.

- $\mathcal{S}(x): \leftrightarrow \exists z \forall S \leq x\left(\square_{\alpha,(x)} S \rightarrow \operatorname{true}^{z}(S)\right)$. Here the variable ' $S$ ' ranges over $\Sigma_{1}^{0}$-sentences.

- $\triangle_{\theta} A: \leftrightarrow \exists x\left(\square_{\theta, x} A \wedge \mathcal{S}(x)\right)$. We will usually write $\triangle$ for $\triangle_{\theta}$ suppressing the contextually given $\theta$. We note that modulo some rewriting $\triangle_{\theta}$ is $\Sigma_{1}^{0}$.

The definition of $\Delta_{\theta}$ is in essence due to Fedor Pakhomov.

As explained in Sect. 2.2, we assume that we have a reasonable coding of proofs in which the code of the proof is larger than the code of the conclusion. We fix, for the moment $\theta$ in the background.

We note that our definition of $\mathcal{S}$ is slightly different from the one in Sect. 4. This is just to compensate for the lack of $\Sigma_{1}^{0}$-collection. We have: 


$$
\begin{aligned}
& \text { EA } \vdash \mathcal{S}(x) \rightarrow \forall S\left(\square_{\alpha,(x)} S \rightarrow \operatorname{true}(S)\right) \\
& \mathrm{EA}+\mathrm{B} \Sigma_{1} \quad \vdash \quad \mathcal{S}(x) \leftrightarrow \forall S\left(\square_{\alpha,(x)} S \rightarrow \operatorname{true}(S)\right) \\
& \text { EA } \vdash \triangle A \rightarrow \exists x\left(\square_{x} A \wedge \forall S\left(\square_{\alpha,(x)} S \rightarrow \operatorname{true}(S)\right)\right) \\
& \mathrm{EA}+\mathrm{B} \Sigma_{1} \vdash \triangle A \leftrightarrow \exists x\left(\square_{x} A \wedge \forall S\left(\square_{\alpha,(x)} S \rightarrow \operatorname{true}(S)\right)\right)
\end{aligned}
$$

We note that we can write the right-hand-side of $(\dagger)$ as:

$$
\left(\exists x \square_{x} A\right)<\left(\exists x \exists S\left(\square_{\alpha,(x)} S \wedge \neg \operatorname{true}(S)\right)\right) .
$$

Here the witness comparison is only concerned with the outer quantifiers.

Lemma $1 \mathrm{EA}+\forall x\left(\square_{\alpha} \square_{x} A \rightarrow \square_{\alpha} A\right) \vdash \square_{\alpha} \triangle A \rightarrow \square_{\alpha} A$.

Proof We find $R$ such that EA $\vdash R \leftrightarrow\left(\exists x \square_{x} A\right)<\square_{\alpha} R$. We note that $R$ is $\Sigma_{1}^{0}$.

We reason in EA $+\forall x\left(\square_{\alpha} \square_{x} A \rightarrow \square_{\alpha} A\right)$. Suppose $\square_{\alpha} \triangle A$. We prove $\square_{\alpha} A$.

We reason inside $\square_{\alpha}$. Since, by assumption, $\triangle A$, we have, for some $x$, (i) $\square_{x} A$ and (ii) $\forall S \leq x\left(\square_{\alpha,(x)} S \rightarrow \operatorname{true}(S)\right)$. In case not $\square_{\alpha,(x)} R$, by (i), we find $R$. If we do have $\square_{\alpha,(x)} R$, we find $R$ by (ii). ${ }^{9}$ We leave the $\square_{\alpha}$-environment.

We have shown $\square_{\alpha} R$. It follows, (a) that for some $p$, we have $\square_{\alpha} \square_{\alpha,(p)} R$ and, by the fixed point equation for $R$, (b) $\square_{\alpha}\left(\left(\exists x \square_{x} A\right)<\square_{\alpha} R\right)$. Combining (a) and (b) and the upward persistence of $\square_{x}$, we find $\square_{\alpha} \square_{p} A$, and, thus, $\square_{\alpha} A$, as desired. We leave $\mathrm{EA}+\forall x\left(\square_{\alpha} \square_{x} A \rightarrow \square_{\alpha} A\right)$.

We have shown EA $+\forall x\left(\square_{\alpha} \square_{x} A \rightarrow \square_{\alpha} A\right) \vdash \square_{\alpha} \triangle A \rightarrow \square_{\alpha} A$.

The proof of Lemma 1 deserves a few comments.

Remark 4 We note that the argument also works when we define $\triangle A$ as $\exists x\left(\square_{x} A \wedge\right.$ $\left.\forall S\left(\square_{\alpha,(x)} S \rightarrow \operatorname{true}(S)\right)\right)$. The argument does not use that $\Delta$ is $\Sigma_{1}^{0}$.

Remark 5 In all applications of Lemma 1, EA verifies not just $\forall x\left(\square_{\alpha} \square_{x} A \rightarrow \square_{\alpha} A\right.$ ) for the concrete choice of $\square$ of the application, but the stronger $\forall x \square_{\alpha}\left(\square_{x} A \rightarrow A\right)$. We note that using this last condition, we may obtain the theorem without the demand that $\square_{y}$ is upward persistent in $y$. In EA $+\forall x \square_{\alpha}\left(\square_{x} A \rightarrow A\right.$ ), we can go from $\square_{\alpha} \square_{\alpha,(p)} R$ and $\square_{\alpha}\left(\left(\exists x \square_{x} A\right)<\square_{\alpha} R\right)$ to $\square_{\alpha} \bigvee_{z<p} \square_{z} A$, and, hence, $\square_{\alpha} A$.

Remark 6 The proof of Lemma 1 does not use exponentiation and would work in $S_{2}^{1}$. The reason is that we only use $\operatorname{true}(R) \rightarrow R$, which is the direction of $\operatorname{true}(R) \leftrightarrow R$ that does not require exponentiation.

Remark 7 Let i-EA be the constructive version of EA. Let $U$ be a constructive theory that extends i-EA. Suppose i-EA $\vdash\left(\theta(y, z) \wedge y<y^{\prime}\right) \rightarrow \theta\left(y^{\prime}, z\right)$.

Then, inspection shows that the entire proof of Lemma 1 also works when we substitute i-EA for EA. This uses the basic insight that $\square_{\alpha,(x)} R$ is decidable in i-EA. So the case-splitting in the proof can be constructively justified.

Thus, we find i-EA $+\forall x\left(\square_{\alpha} \square_{x} A \rightarrow \square_{\alpha} A\right) \vdash \square_{\alpha} \triangle A \rightarrow \square_{\alpha} A$.

\footnotetext{
9 We note that, in this step, we use that (the code of) the conclusion is smaller than (the code of) the proof.
} 
We prove the outside-big-inside-small lemma for $\mathcal{S}$ as a notion of smallness in EA. The proof has to be a bit more elaborate that in the luxurious case where we had full PA to work with.

Lemma 2 EA $\vdash \forall x \square_{\alpha} \mathcal{S}(x)$.

Proof We work in EA. We prove the desired result by induction on $x$. We need a multiexponential bound for the $\square_{\alpha}$-proofs. We will extract the desired bound by inspecting the induction step.

The base case is trivial since there will be no $S \leq 0$. The proof witnessing the base will be given by a standard number $\underline{n}$.

We turn to the induction step. Suppose $p_{0}$ witnesses $\square_{\alpha} \mathcal{S}(x)$. We have two possibilities: $\operatorname{proof}_{\alpha}\left(x+1, S^{*}\right)$, for some $S^{*} \leq x+1$, or $\neg \operatorname{proof}_{\alpha}\left(x+1, S^{*}\right)$, for all $S^{*} \leq x+1$.

Suppose $\operatorname{proof}_{\alpha}\left(x+1, S^{*}\right)$. Inspecting the proof of the truth-lemma for true in [10, Ch. V, Section 5b, pp. 361-366], we obtain a $p_{1}$ such that $\operatorname{proof}_{\alpha}\left(p_{1}, S^{*} \rightarrow \operatorname{true}\left(S^{*}\right)\right)$. The transformation $S^{*} \mapsto p_{1}$ is p-time. By [10, Ch. III, Lemma 3.14, p. 175], we obtain an $\alpha$-proof $p_{2}$ of $\operatorname{proof}_{\alpha}\left(x+1, S^{*}\right)$. The transformation $x+1 \mapsto p_{2}$ is of order $2_{k}^{x+1}$, where $k$ is standard and the subscript $\underline{k}$ indicates the number of iterations of exponentiation. Working inside $\square_{\alpha}$ we can put these facts together to obtain

$$
\text { (a) } \mathcal{S}(x), \quad \text { (b) } \operatorname{proof}_{\alpha}\left(x+1, S^{*}\right) \text { and (c) } \operatorname{true}\left(S^{*}\right) \text {. }
$$

Let $z_{0}$ be the witness of (a), let $z_{1}$ be the witness of (c). Then, it is easily seen that $z:=\max \left(z_{0}, z_{1}\right)$ witnesses $\mathcal{S}(x+1)$.

Suppose $\forall S^{*} \leq x+1 \neg \operatorname{proof}_{\alpha}\left(x+1, S^{*}\right)$. By [10, Ch. III, Lemma 3.14, p. 175], we may find an $\alpha$-proof $p_{3}$ of $\forall S^{*} \leq x+1 \neg \operatorname{proof}_{\alpha}\left(x+1, S^{*}\right)$ where the transformation $x+1 \mapsto p_{3}$ is of order $2_{\underline{k}}^{x+1}$. Using

$$
\text { (d) } \forall S^{*} \leq x+1 \neg \operatorname{proof}_{\alpha}\left(x+1, S^{*}\right)
$$

inside $\square_{\alpha}$, we easily find the desired proof of $\mathcal{S}(x+1)$.

We note that apart from a bit of overhead we extend $p_{0}$ with at most two proofs that are estimated by $2_{k}^{x+1}$. So, the resulting proof will be of order $p_{0} \times\left(2_{k}^{x+1}\right)^{2}$. Thus, after all is said and done, the proof we obtain of $\mathcal{S}(x+1)$ will be estimated by $\underline{n} \times\left(2_{\underline{k}}^{x+1}\right)^{2(x+1)}=2^{2^{x+1} \times 2(x+1)} \leq 2_{\underline{k}}^{2 x+1}$, assuming that $k \geq 2$.

Lemma 3 EA $\vdash \forall x \square_{\alpha}\left(\square_{x} A \rightarrow \triangle A\right)$. Hence,

$$
\mathrm{EA}+\left(\square_{\alpha} A \rightarrow \exists x \square_{\alpha} \square_{x} A\right) \vdash \square_{\alpha} A \rightarrow \square_{\alpha} \triangle A
$$

Proof We work in EA. Let $x$ be given. By Lemma 2, we find $\square_{\alpha} \mathcal{S}(x)$. Thus, $\square_{\alpha}\left(\square_{x} A \rightarrow\left(\square_{x} A \wedge \mathcal{S}(x)\right)\right)$. This gives us $\square_{\alpha}\left(\square_{x} A \rightarrow \triangle A\right)$. 
Lemma $4 \mathrm{EA}+\forall x\left(\left(\square_{x} A \wedge \square_{x}(A \rightarrow B)\right) \rightarrow \square_{x} B\right) \vdash(\triangle A \wedge \triangle(A \rightarrow B)) \rightarrow \triangle B$.

Proof We work in EA $+\forall x\left(\left(\square_{x} A \wedge \square_{x}(A \rightarrow B)\right) \rightarrow \square_{x} B\right)$. Suppose $\triangle A$ and $\triangle(A \rightarrow B)$. It follows that, for some $x$, we have $\square_{x} A$ and $\mathcal{S}(x)$ and that, for some $y$, we have $\square_{y}(A \rightarrow B)$ and $\mathcal{S}(y)$. Let $z:=\max (x, y)$. It is easily seen that $\square_{z} A$ and $\square_{z}(A \rightarrow B)$ and $\mathcal{S}(z)$. Hence, $\square_{z} B$ and $\mathcal{S}(z)$, and, thus, $\triangle B$.

Lemma $5 \mathrm{EA}+\forall S \exists x \square_{\alpha}\left(S \rightarrow \square_{x} S\right) \vdash \square_{\alpha}(S \rightarrow \triangle S)$.

Proof This is immediate by Lemma 2.

We formulate the obvious theorem that follows from the Lemmas. Let $\mathrm{W}_{\alpha, \theta}$ be $\mathrm{EA}$ plus the following principles:
a. $\forall x\left(\square_{\alpha} \square_{x} A \rightarrow \square_{\alpha} A\right)$
b. $\square_{\alpha} A \rightarrow \exists x \square_{\alpha} \square_{x} A$
c. $\forall x\left(\left(\square_{x} A \wedge \square_{x}(A \rightarrow B)\right) \rightarrow \square_{x} B\right)$
d. $\forall S \exists x \square_{\alpha}\left(S \rightarrow \square_{x} S\right)$

Let $\mathrm{W}_{\alpha, \theta}^{+}$be EA plus the following principles.
A. $\square_{\alpha} \triangle A \rightarrow \square_{\alpha} A$
B. $\square_{\alpha} A \rightarrow \square_{\alpha} \triangle A$
C. $(\triangle A \wedge \triangle(A \rightarrow B)) \rightarrow \triangle B$
D. $\forall S \square_{\alpha}(S \rightarrow \triangle S)$

Theorem 13 Let $\alpha$ be a $\Delta_{0}(\mathrm{exp})$-predicate that numerates the axioms of $U$ in $\mathrm{EA}$, or, equivalently, in true arithmetic. Let $\theta$ be a $\Sigma_{1}^{0}$-predicate that satisfies $\mathrm{EA} \vdash(\theta(y, z) \wedge$ $\left.y<y^{\prime}\right) \rightarrow \theta\left(y^{\prime}, z\right)$. Then, $\mathrm{W}_{\alpha, \theta}$ implies $\mathrm{W}_{\alpha, \theta}^{+}$.

The logic GLT is the bimodal propositional logic which has GL both for $\square$ and $\triangle$, plus the following principles.

- $\vdash \Delta \phi \rightarrow \square \phi$.

- $\vdash \square \phi \rightarrow \triangle \square \phi$.

- $\vdash \square \phi \rightarrow \square \triangle \phi$.

- $\vdash \square \Delta \phi \rightarrow \square \phi$.

By Theorem 13, we have:

Theorem 14 Let $\alpha$ be a $\Delta_{0}(\exp )$-predicate that numerates the axioms of $U$ in $E A$, or, equivalently, in true arithmetic. Let $\theta$ be a $\Sigma_{1}^{0}$-predicate that satisfies $\mathrm{EA} \vdash(\theta(y, z) \wedge$ $\left.y<y^{\prime}\right) \rightarrow \theta\left(y^{\prime}, z\right)$.

Suppose $\mathrm{W}_{\alpha, \theta}$ is a true theory and $U \vdash \mathrm{W}_{\alpha, \theta}$. Then GLT is arithmetically valid in $U$. In addition, $U$ satisfies $\mathrm{HB}$ both for $\square_{\alpha}$ and for $\triangle_{\theta}$. Finally, $\triangle_{\theta}$ satisfies the Kreisel Condition in $U$.

\subsection{Extensions of Peano arithmetic revisited}

We show how the case of extensions of Peano Arithmetic, treated in Sect. 4, fits the framework of the present section. 
Let $U$ be a consistent extension of PA and let $\alpha$ be an elementary predicate numerating the axioms of $U$ in EA with $\pi \preceq \alpha$. We note that, equivalently, $\alpha$ numerates the axioms of $U$ in true arithmetic. Let $\alpha_{z}(x): \leftrightarrow \alpha(x) \wedge x \leq z$. We take $\Theta_{\alpha}(z, x):=\operatorname{prov}_{\alpha_{z}}(x)$ in the role of $\theta$. Thus, we have $\square_{\alpha_{z}} A=\Theta_{\alpha}(z,\ulcorner A\urcorner)$ in the role of $\square_{z} A$ and we have $\widetilde{\square}_{\alpha} A:=\exists z\left(\square_{\alpha_{z}} A \wedge \mathcal{S}(z)\right)$ the role of $\triangle A$.

We define: $\widetilde{\alpha}(a): \leftrightarrow \alpha(a) \wedge \mathcal{S}(a)$. We have:

Lemma 6 EA $\vdash \forall A\left(\widetilde{\square}_{\alpha} A \leftrightarrow \square \widetilde{\alpha} A\right)$.

Proof We reason in EA.

Suppose $\widetilde{\square}_{\alpha} A$. Then, for some $z$, we have $\square_{\alpha_{z}} A$ and $\mathcal{S}(z)$. Suppose $p$ witnesses $\square_{\alpha_{z}} A$ and $B$ is an axiom used in $p$. Then, $\alpha(B)$ and $B \leq z$. Since $\mathcal{S}$ is downward persistent w.r.t. $\leq$, we find $\mathcal{S}(B)$, and, hence, $\widetilde{\alpha}(B)$.

Conversely, suppose $\square_{\widetilde{\alpha}} A$. let $q$ be a witnessing proof. Let $B$ be the maximal $\alpha$-axiom used in $q$. We find $\mathcal{S}(B)$. Thus, $\square_{\alpha_{B}} A$ and $\mathcal{S}(B)$, i.e., $\widetilde{\square}_{\alpha} A$.

Lemma 7 The predicate $\tilde{\alpha}$ numerates the axioms of $U$ in $U$. Hence, $\widetilde{\square}_{\alpha}$ is Fefermanian in $U$ over $U$.

Proof Let $X$ be the set of axioms set of axioms numerated by $\alpha$.

Suppose $n \in X$. Then $\alpha(n)$ and hence $U \vdash \alpha(\underline{n})$. Since also, by Lemma 2, we have $U \vdash \mathcal{S}(\underline{n})$, it follows that $U \vdash \widetilde{\alpha}(\underline{n})$.

Suppose $U \vdash \widetilde{\alpha}(\underline{n})$. Then, $U \vdash \alpha(\underline{n})$ and, hence, $n \in X$.

Lemma 8 EA verifies $\mathrm{W}_{\alpha, \Theta_{\alpha}}$.

Proof The principle (a) follows by essential reflexivity. The principles (b) and (c) are trivial. The principle (d) follows since for a sufficiently large $n$ we will have, in EA, that $\square_{\alpha_{\underline{n}}} B$, where $B$ is a single axiom for EA.

By Theorem 14 and Lemma 8 we find:

Theorem 15 The logic GLT is arithmetically valid for $\square_{\alpha}$ and for $\square_{\widetilde{\alpha}}$ over $U$. In addition, we have HB both for $\square_{\alpha}$ and for $\square \widetilde{\alpha}$ over U. Finally, $\square_{\widetilde{\alpha}}$ satisfies the Kreisel Condition in $U$.

In case $U$ is sound, one easily sees that the pair $\square_{\alpha}$ and $\square_{\widetilde{\alpha}}$ satisfies the conditions of Theorem 16 of [11]. It follows that GLT is precisely the bi-modal propositional logic of $\square_{\alpha}$ and $\square \widetilde{\alpha}$ in $U$, for sound $U$.

Remark 8 Let EA + ref be EA plus sentential reflection for predicate logic. Let $\tau$ be a standard axiomatization for EA + ref. Let $U$ be an extension of EA + ref and let $\alpha$ be an elementary axiomatization of $U$ such that $\tau \preceq \alpha$. With these basic ingredients we can repeat the development of the present section noting that we are always looking at sentential reflection rather than uniform reflection.

In [31] we introduced the theory Peano Corto, which has many analogies to EA+ref. It would be interesting to see how much of our development can be repeated for the case of Peano Corto. 


\section{Extensions of elementary arithmetic}

In this section, we give a general construction of a $\theta$ with the desired properties for extensions of EA.

We first take a moment to see that, in order to get the desired combinations of properties for extensions of EA, we indeed need to leave the realm of the Fefermanian predicates.

\subsection{Two examples}

Our first example addresses the case that we only demand that our Fefermanian predicate is Kreiselian.

Example 2 Consider the theory $U:=\mathrm{EA}+\square_{\beta} \perp$. Here $\beta$ numerates a single axiom for EA. Suppose there would be a Fefermanian predicate for $U$ over EA that is Kreiselian. Say the witnessing predicate for the axiom set is $\alpha$. Let $\gamma(x): \leftrightarrow \beta(x) \vee x=\left\ulcorner\square_{\beta} \perp\right\urcorner$. We have $\gamma \preceq \alpha$. Since, $U \vdash \square_{\beta} \perp$, it follows that $U \vdash \square_{\gamma} \perp$, and, hence, $U \overline{\vdash \square_{\alpha} \perp \text {. }}$ So, $U \vdash \perp$. Quod non.

In the previous example, we needed an unsound theory. In our second example, we consider the case that our example satisfies absorption. Here we can use a sound theory.

Example 3 Let $U:=$ EA. Consider a Fefermanian predicate $P$ based on $\alpha$ for EA over EA. We write $\triangle$ for $P$. We note that $\beta \preceq \alpha$. Suppose we would have the absorption law for $\triangle$ and $\square_{\beta}$. Then, it would follow that:

$$
\begin{aligned}
\mathrm{EA} \vdash \square_{\beta} \square_{\beta} \perp & \rightarrow \square_{\beta} \triangle \perp \\
& \rightarrow \square_{\beta} \perp
\end{aligned}
$$

So, by Löb's Rule, EA $\vdash \square_{\beta} \perp$. Quod non.

Question 1 We note that our examples are of finitely axiomatized theories. The construction of Sect. 5.2 gives us Fefermanian predicates for theories extending Peano Arithmetic. As pointed out in Remark 8, we can improve this to extensions of EA+ref. Obviously there is a big gap between our examples and counterexamples for the possibility to obtain a Fefermanian $\triangle$.

So, the question is whether we can find a larger class of theories for which we have a Fefermanian $\triangle$ that satisfies both the Löb Conditions and the Kreisel Condition and that satisfies the emission and absorption laws.

\subsection{Motivating remarks for our construction}

We may construct the desired predicates $\square_{x}$ in many ways. However, for didactic reasons, it good to maximize the meaningfulness of the construction.

As a first step, we note that we have the conditions of Theorems 1 and 3 , for $\square_{\alpha,(x)}$. So, to obtain absorption and emission, $\square_{x}:=\square_{\alpha,(x)}$ is already sufficient. The idea of 
our construction is simply to add closure under modus ponens and closure under HB in a minimal way to $\square_{\alpha,(x)}$.

The minimal way to obtain the addition of modus ponens is simply to close of the $\alpha$-theorems with proofs $\leq x$ under modus ponens. However, we can strengthen the analogy with our approach to the case of extensions of PA by working with a Hilbert system that only has modus ponens as a rule. Such deduction systems are described in [25] (first edition 1940) and in [3]. When we have such a system we can, for the definition of $\square_{x}$, consider the theorems whose proofs contain only axioms whether logical or non-logical which are $\leq x$. Thus, the main difference between our approach for the extensions of PA and the new one is that we stop treating logical and non-logical axioms as different.

What to do to obtain the Hilbert-Bernays condition? Simple: we add the true $\Sigma_{1}^{0}$ sentences to our original axiom set.

There is a small technical complication, due to the lack of $\Sigma_{1}^{0}$-collection, that necessitates us to stipulate a bound on the witnesses of the truth of the $\Sigma_{1}^{0}$ sentences involved in a proof, but this complication disappears as soon as we have $\Sigma_{1}^{0}$-collection in the ambient theory.

\subsection{The construction}

We fix a Hilbert system $\mathfrak{H}$ with as only rule modus ponens. Let logic $(x)$ be a $\Delta_{0}(\exp )$ formula that numerates the set axioms of $\mathfrak{H}$ in EA.

We assume that a $\Sigma_{1}^{0}$-sentence begins with a, possibly vacuous, existential quantifier.

We give the basic definitions for our approach. Let a theory $U$ be given and a $\Delta_{0}(\exp )$-formula $\alpha$ that numerates the axioms of $U$ in EA (or, equivalently, in true arithmetic).

- We define $\operatorname{ass}^{\circ}(p)$ as the set of assumptions op $p$, where now a logical axiom also counts as an assumption. In other words, anything not proved from previous items using modus ponens counts as an assumption.

- We write $\operatorname{proof}_{\gamma}^{\circ}(p, x)$ for $\operatorname{proof}(p, x) \wedge \forall y \in \operatorname{ass}^{\circ}(p) \gamma(y)$.

- We write $\square_{\gamma} A$ for $\exists p \operatorname{proof}_{\gamma}^{\circ}(p,\ulcorner A\urcorner)$.

- $B$ is a direct o-subformula of $A$ if $A$ is of the form $(C \rightarrow B)$ or $(B \rightarrow C)$. The o-subformulas of $A$ are the smallest set that contains $A$ and is closed under taking direct o-subformulas.

- $\alpha^{+}(a):=\alpha(a) \vee \operatorname{logic}(a) \vee \operatorname{true}(a)$.

Here true is a $\Sigma_{1}^{0}$-truth predicate. We we take true $(a)$ to imply that $a$ is a $\Sigma_{1}^{0}$ sentence.

- $\widetilde{\alpha}^{+}(a):=\alpha^{+}(a) \wedge \mathcal{S}(a)$.

- $\alpha_{x, z}^{+}(a): \leftrightarrow\left(\alpha(a) \vee \operatorname{logic}(a) \vee \operatorname{true}^{z}(a)\right) \wedge a \leq x$.

- $\alpha_{x}^{+}(a): \leftrightarrow \alpha^{+}(a) \wedge a \leq x$.

- $\Theta_{\alpha}^{\circ}(x, A): \leftrightarrow \square_{\alpha_{x, *}^{+}} A: \leftrightarrow \exists z \square_{\alpha_{x, z}^{+}} A$.

We use $\Theta_{\alpha}^{\circ}$ in the role of $\theta$. So $\square_{\alpha_{x, *}^{+}}(\cdot)$ has the role of $\square_{x}$.

- We define $\widetilde{\varpi}_{\alpha^{+}} A: \leftrightarrow \exists x\left(\square_{\alpha_{x, *}^{+}} A \wedge \mathcal{S}(x)\right)$. So, $\widetilde{\varpi}_{\alpha^{+}}$has the role of $\triangle$. 
In case we have $\Sigma_{1}^{0}$-collection, the situation simplifies. We note that in the absence of $\Sigma_{1}^{0}$-collection $\square_{\alpha_{x}^{+}} A$ is not $\Sigma_{1}^{0}$ but $\Sigma_{1,1}^{0}$. See [31] or [32] for an explanation of $\Sigma_{1,1}^{0}$. We have:

Lemma 9 a. EA $\vdash \square_{\alpha_{x, *}^{+}} A \rightarrow \square_{\alpha_{x}^{+}} A$.

b. $\mathrm{EA}+\mathrm{B} \Sigma_{1} \vdash \square_{\alpha_{x}^{+}} A \leftrightarrow \square_{\alpha_{x, *}^{+}} A$.

c. $\mathrm{EA} \vdash \widetilde{\square}_{\alpha^{+}} A \rightarrow \square_{\tilde{\alpha}^{+}} A$.

d. $\mathrm{EA}+\mathrm{B} \Sigma_{1} \vdash \widetilde{\alpha}_{\widetilde{\alpha}^{+}} A \leftrightarrow \widetilde{\varpi}_{\alpha^{+}} A$.

Proof (a) is trivial. (b) is an immediate application of collection. (c) and (d) are analogous to the proof of Lemma 6, using respectively (a) and (b).

We start with a well-known lemma.

Lemma 10 Let $\alpha$ be a $\Delta_{0}(\exp )$-predicate numerating the axiom set of $U$ over EA. Then, EA $\vdash \forall x, A \square_{\alpha}\left(\square_{\alpha,(x)} A \rightarrow A\right)$.

Proof We reason in EA. Suppose, for some $p \leq x$, we have $\operatorname{proof}_{\alpha}(p, A)$. It clearly follows that $\square_{\alpha} A$ and, hence, a fortiori, $\square_{\alpha}\left(\square_{\alpha,(x)} A \rightarrow A\right)$.

Suppose, for all $q \leq x$, we have $\neg \operatorname{proof}_{\alpha}(q, A)$. If follows, by $\Sigma_{1}^{0}$-completeness, that $\square_{\alpha} \forall q \leq p \neg \operatorname{proof}_{\alpha}(q, A)$. In other words, $\square_{\alpha} \neg \square_{\alpha,(x)} A$. If follows that $\square_{\alpha}\left(\square_{\alpha,(x)} A \rightarrow A\right)$.

The next lemma is in the spirit of the previous one, but takes a bit more work.

Lemma 11 Let $\alpha$ be a $\Delta_{0}(\exp )$-predicate numerating the axiom set of $U$ over $E A$. We have $\mathrm{EA} \vdash \forall x, A \square_{\alpha}\left(\square_{\alpha_{x, *}^{+}} A \rightarrow A\right)$.

Proof We will use a well-known fact, to wit that

$$
\mathrm{EA} \vdash \forall x \square_{\alpha} \forall y\left(y \leq x \leftrightarrow \bigvee_{z \leq x} z=y\right)
$$

This fact means that we do not have to worry that undesirable non-standard elements creep in below elements that are internally standard in EA.

We reason in EA. Let $x$ be given.

We reason inside $\square_{\alpha}$. Suppose (\$) $\square_{\alpha_{x, *}^{+}} A$. Let $z$ and $p$ witness $\square_{\alpha_{x, *}^{+}} A$. Keeping $z$ fixed, we may, by the $\Delta_{0}$ (exp)-mimimum Principle, find a $p_{0}$ that is minimal with this property.

Suppose that $p_{0}$ contains a formula $B$ twice. If $B$ is the conclusion $A$ of $p_{0}$ we may omit the part after the first occurrence of $A$, obtaining a shorter proof. This contradicts the minimality of $p_{0}$. If $B$ is not the conclusion of $p_{0}$, we may omit all occurrences of $B$ after the first one, obtaining a shorter proof. This again contradicts the minimality of $p_{0}$. We may conclude that all sentences in $p_{0}$ occur only once in $p_{0}$.

We claim that every formula that is a (sub)conclusion of $p_{0}$ is a o-subformula of a formula in ass $^{\circ}\left(p_{0}\right)$. Suppose not. Let $B$ be the first such formula. Clearly, $B$ cannot be a o-assumption. So, it must be the conclusion of an application of modus ponens 
and, thus, a direct o-subformula of a previous formula of the form $(C \rightarrow B)$. But this formula is by assumption a o-subformula of $\operatorname{ass}^{\circ}\left(p_{0}\right)$. A contradiction.

So, all sentences occurring in $p_{0}$ are in o-subformulas of $\operatorname{ass}^{\circ}\left(p_{0}\right)$ and occur only once. It follows that the sentences in $p_{0}$ are all $\leq x$ and, hence, the number of these sentences is also $\leq x$. So, by our assumptions on coding, we find $p_{0} \approx x^{x}$. So, certainly $p_{0}$ will be estimated by $2^{x^{2}}+\underline{k}$, for a sufficiently large standard $k$.

It follows that $\bigvee_{q \leq 2^{x^{2}+\underline{k}}} \operatorname{proof}_{\alpha^{+}}^{\circ}(q, A)$ and, hence,

$$
(\dagger) \bigvee_{q \leq 2^{x^{2}}+\underline{k}} \operatorname{proof}_{\alpha \cup \operatorname{true}}(q, A) \text {. }
$$

(Here the $q$ are standard on the $\square_{\alpha}$-external EA-level.)

Now, suppose $(\ddagger)$ proof $_{\alpha \cup \text { true }}(q, A)$, where $q \leq 2^{x^{2}}+\underline{k}$. We transform $q$ as follows. Let $\mathscr{S}$ be the set of the $\Sigma_{1}^{0}$-sentences in ass $(q)$ that are not in $\alpha$. It follows that all $S \in \mathscr{S}$ are true. We transform $q$ in two steps. First we form a proof $q^{\prime}$ from the assumptions $(\operatorname{ass}(q) \backslash \mathscr{S}) \cup \bigwedge \mathscr{S}$ with conclusion $A$. Then, we transform $q^{\prime}$ to $q^{\prime \prime}$ with assumptions $\operatorname{ass}(q) \backslash \mathscr{S}$ to $\bigwedge \mathscr{S} \rightarrow A$. We note that the big conjunction is bounded by $q$ and, thus, exists at the $\square_{\alpha}$-external level.

We easily see that $\left|q^{\prime}\right|$ can be bounded by a linear term in $|q|$. The transformation $q^{\prime} \mapsto q^{\prime \prime}$ uses the deduction theorem. Inspection of the proof shows that here also $\left|q^{\prime \prime}\right|$ is linear in $\left|q^{\prime}\right|$. Thus, $q^{\prime \prime}$ is bounded by $2^{\underline{m}} x^{2}+\underline{n}$, for appropriate standard $m$ and $n$. We conclude that $q^{\prime \prime}$ is also $\square_{\alpha}$-external. We have found that $\square_{\alpha,\left(q^{\prime \prime}\right)}(\bigwedge \mathscr{S} \rightarrow A)$, where $q^{\prime \prime}$ is $\square_{\alpha}$-external.

We apply Lemma 10 to obtain $\bigwedge \mathscr{S} \rightarrow A$. We also have, since all elements of $\mathcal{S}$ are true and $\mathcal{S}$ is $\square_{\alpha}$-external, that $\bigwedge\{\operatorname{true}(S) \mid S \in \mathscr{S}\}$. From this it follows that $\bigwedge \mathcal{S}$. Combining $\bigwedge \mathscr{S} \rightarrow A$ and $\bigwedge \mathcal{S}$, we find $A$.

By $(\dagger)$ we find $A$ without assumption ( $\ddagger)$. We now cancel (\$) to obtain the sentence: $\square_{\alpha_{x, *}^{+}} A \rightarrow A$.

We leave the $\square_{\alpha}$-environment. We have shown $\square_{\alpha}\left(\square_{\alpha_{x, *}^{+}} A \rightarrow A\right)$, as desired.

We insert a quick corollary of Lemma 11.

Corollary 1 Let $\alpha$ be a $\Delta_{0}(\mathrm{exp})$-predicate numerating the axiom set of $U$ over EA. Then, EA $\vdash \forall A\left(\exists x \square_{\alpha_{x, *}^{+}} A \leftrightarrow \square_{\alpha} A\right)$.

Proof We reason in EA. The left-to-right direction works as follows. We use Lemma 11.

$$
\begin{aligned}
\exists x \square_{\alpha_{x, *}^{+}} A & \rightarrow \exists x \square_{\alpha} \square_{\alpha_{x, *}^{+}} A \\
& \rightarrow \square_{\alpha} A
\end{aligned}
$$

The right-to-left direction is immediate since $\square_{\alpha} A$ implies $\square_{\alpha,(x)} A$, for some $x$, and $\square_{\alpha,(x)} A$ implies $\square_{\alpha_{x, *}^{+}} A$.

Lemma 12 Let $\alpha$ be a $\Delta_{0}(\mathrm{exp})$-predicate numerating the axiom set of $U$ over $\mathrm{EA}$.

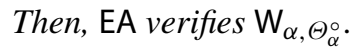


Proof The principle (a) follows by Lemma 11. The principle (b) follows by:

$$
\begin{aligned}
\mathrm{EA} \vdash \square_{\alpha} A & \rightarrow \exists x \square_{\alpha}\left(\square_{\alpha,(x)} A \wedge \mathcal{S}(x)\right) \\
& \rightarrow \exists x \square_{\alpha}\left(\square_{\alpha_{x, *}^{+}} A \wedge \mathcal{S}(x)\right)
\end{aligned}
$$

The principles (c) and (d) are immediate by the construction of $\square_{\alpha_{x, *}^{+}}$.

By Theorem 14 and Lemma 12 we find:

Theorem 16 Let $\alpha$ be a $\Delta_{0}(\exp )$-predicate numerating the axiom set of $U$ over EA. Then, the logic GLT is arithmetically valid in $U$ for $\widetilde{\square}_{\alpha^{+}}$and $\square_{\alpha}$. In addition, we have HB over $U$ both for $\square_{\alpha}$ and for $\square_{\widetilde{\alpha}}$. Finally, $\widetilde{\square}_{\alpha^{+}}$satisfies the Kreisel Condition in $U$.

Question 2 The predicate $\square_{\alpha_{x, *}^{+}}$allows us to give an Orey Hájek Characterization for extensions of EA. Suppose $\gamma$ is an elementary predicate that numerates the axioms of $V$ over EA. Then, $U$ is $\Pi_{1}^{0}$-conservative over $V$ iff, for all $n$, we have $U \vdash \diamond_{\gamma_{n, *}^{+}} \top$.

It seems to me that, using this characterization, many results in the work of Per Lindström and Christian Bennet should be transferable from the case of extensions of PA to the case of extensions of EA. It would be interesting to explore this.

Question 3 In our definition of $\square$, we counted instances of all proposition-logical and predicate-logical schemes as axioms. I think we only need to count the instances of a few specific schemes that are essentially predicate logical. It would be interesting to explore this.

\section{An application}

We first prove a very general result.

Theorem 17 Let $U$ be any theory and suppose that $\triangle$ satisfies the Löb Conditions in $U$ and $\mathrm{HB}$, to wit, $U \vdash S \rightarrow \triangle S$, for $S \in \Sigma_{1}^{0} \cdot{ }^{10}$ We have:

i. $U$ is $\Pi_{1}^{0}$-conservative over $U+\triangle \perp$.

ii. If $\triangle$ satisfies the Kreisel condition for $U$, then $U$ is $\Sigma_{1}^{0}$-conservative over $U+\neg \triangle \perp$.

Proof Suppose $\triangle$ satisfies the Löb conditions and the HB for $U$.

We prove (i). Let $P$ be a $\Pi_{1}^{0}$-sentence. Suppose $U+\triangle \perp \vdash P$. Then, (a) $U+\neg P \vdash$ $\neg \triangle \perp$. Hence, $U \vdash \triangle \neg P \rightarrow \triangle \neg \triangle \perp$. It follows by HB and by the formalized Second Incompleteness Theorem for $\triangle$, which follows by the Löb Conditions, that (b) $U+\neg P \vdash \triangle \perp$. Combining (a) and (b), we find $U \vdash P$.

We prove (ii). Suppose that $\triangle$ satisfies the Kreisel Condition for $U$. Let $S$ be a $\Sigma_{1}^{0}$-sentence. Suppose $U+\neg \triangle \perp \vdash S$. It follows that $U \vdash \triangle \perp \vee S$, and, hence, by the Löb Conditions and $\mathrm{HB}, U \vdash \triangle S$. By the Kreisel Condition, we find $U \vdash S$.

The proof of (i) is ascribed by Per Lindström, in [20, p94], to Georg Kreisel in [17].

10 We note that in the present general context the Löb Conditions and HB are mutually independent. 
Consider any recursively enumerable theory $U$ and let $\alpha$ be a $\Delta_{0}(\exp )$-formula that numerates a set of axioms for $U$ in EA. We note that, by Craig's trick, we can always find such a $\Delta_{0}$ (exp)-formula. We take $\Delta_{\alpha}:=\widetilde{\emptyset}_{\alpha^{+}}$. We note that $\Delta$ fulfills the conditions of Theorem 17 for $U$. It follows that $\triangle_{\alpha} \perp$ is a $\Sigma_{1}^{0}$-sentence such that $U$ is $\Pi_{1}^{0}$-conservative over $U+\triangle_{\alpha} \perp$ and $\neg \triangle_{\alpha} \perp$ is a $\Pi_{1}^{0}$-sentence such that $U$ is $\Sigma_{1}^{0}$-conservative over $U+\triangle_{\alpha} \perp$.

For extensions $U$ of Peano Arithmetic, the existence of a $\Sigma_{1}^{0}$-sentence $S$, such that $U$ is $\Pi_{1}^{0}$-conservative over $U+S$ and $U$ is $\Sigma_{1}^{0}$-conservative over $U+\neg S$ is a special case of a result due to Robert Solovay. See [8]. See also [20, Chapter 5].

Remark 9 We note that $\triangle_{\alpha} \perp$ is a fortiori a Rosser sentence for $U$. The resulting proof of Rosser's Theorem is like the proof of the Second Incompleteness Theorem in the sense that the sentence under consideration is self-reference-free, but in the proof of the desired property we do use self-reference. Another example of a self-referencefree $\Sigma_{1}^{0}$ Rosser sentence (for extensions of PA) is due to Fedor Pakhomov. See [23]. We note that Pakhomov's construction is, in a sense, orthogonal to ours. An essential feature of Pakhomov's construction is that, like the ordinary Rosser sentence and its opposite, it produces $\Sigma_{1}^{0}$-sentences $S_{0}$ and $S_{1}$, each with the Rosser property over $U$, such that we have $U \vdash \neg\left(S_{0} \wedge S_{1}\right)$ and $U \vdash \square_{\alpha} \perp \leftrightarrow\left(S_{0} \vee S_{1}\right)$. It follows that e.g. $U \vdash S_{0} \rightarrow \neg S_{1}$, but $U \nvdash \neg S_{1}$. So, $S_{0}$ is not $\Pi_{1}^{0}$-conservative. The non- $\Pi_{1}^{0}$ conservativity of Pakhomov's sentences is an important feature since it allows him to use them for his alternative proof of Solovay's arithmetical completeneness theorem for Löb's Logic.

We formulate a consequence of Theorem 11.

Theorem 18 Suppose $U$ is a recursively enumerable extension of PA. Then there is a $\Sigma_{1}^{0}$-predicate $\sigma^{*}$ such that $\left\langle U, U, \sigma^{*}\right\rangle$ is Fefermanian and $\square_{\sigma^{*}}$ satisfies, in $U$, the Löb-conditions and the Kreisel condition.

Proof Consider a recursively enumerable extension $U$ of PA. We can easily construct a $\Sigma_{1}^{0}$-formula $\sigma$ such that $\langle\mathrm{PA}, U, \sigma\rangle$ is Fefermanian and $\pi \preceq \sigma$. We now apply Theorem 11 to obtain the desired $\sigma^{*}$.

It follows from Theorems 17(ii) and 18 that:

Theorem 19 Suppose $U$ is a recursively enumerable extension of PA. Then, there is a $\Sigma_{1}^{0}$-predicate $\sigma^{*}$ such that $\left\langle U, U, \sigma^{*}\right\rangle$ is Fefermanian and $\diamond_{\sigma^{*}} \top$ is $\Sigma_{1}^{0}$-conservative over $U$.

Theorem 19 stands in interesting contrast to a result due to Craig Smoryński that is reported in Example 1.6 of [8].

Theorem 20 (Smoryński). Suppose $U$ is an extension of PA such $\langle\mathrm{PA}, U, \sigma\rangle$ is Fefermanian and $\alpha$ is $\Delta_{1}^{0}(\mathrm{PA})$. Then, $\diamond_{\alpha}^{\top} \top$ is $\Sigma_{1}^{0}$-conservative over $U$ iff $U$ is $\Sigma_{1}^{0}$-sound.

Inspection of the proof shows that no special properties of PA are used (except its $\Sigma_{1}^{0}$-soundness when it occurs in the role of base theory), so, in fact, we have a far more general result. However, we will not pursue that line here. We just present a variation of Smoryński's result for extensions of PA. 
Theorem 21 Suppose $\langle\mathrm{PA}, U, \sigma\rangle$ is Fefermanian, where $\sigma$ is $\Sigma_{1}^{0}$. Then, the following are equivalent.

i. $U$ is $\Sigma_{1}^{0}$-sound.

ii. $\square_{\sigma}$ satisfies the Kreisel condition for $U$.

iii. $\diamond_{\sigma} \top$ is $\Sigma_{1}^{0}$-conservative over $U$.

Proof Suppose $\langle\mathrm{PA}, U, \sigma\rangle$ is Fefermanian, where $\sigma$ is $\Sigma_{1}^{0}$.

(i) $\Rightarrow$ (ii). Suppose $U$ is $\Sigma_{1}^{0}$-sound. We note that the fact that $\langle\mathrm{PA}, U, \sigma\rangle$ is Fefermanian, implies that $\sigma$ truly enumerates the Gödel codes of an axiom set of $U$. Suppose $U \vdash \square_{\sigma} A$. Then, $\square_{\sigma} A$ is true, and, hence $U \vdash A$.

(ii) $\Rightarrow$ (iii). This is immediate by Theorem 17 .

(iii) $\Rightarrow$ (i). Suppose $U+\diamond_{\sigma} \top$ is $\Sigma_{1}^{0}$-conservative over $U$. By Theorem 9, we have $U \vdash \forall A\left(\square_{\hat{\sigma}} A \leftrightarrow \square_{\sigma} A\right)$. So, $U+\diamond_{\hat{\sigma}} \top$ is $\Sigma_{1}^{0}$-conservative over $U$. By Theorem 10, the triple $\langle\mathrm{PA}, U, \hat{\sigma}\rangle$ is Fefermanian. Moreover, $\hat{\sigma}$ is elementary and, hence, a fortiori, $\Delta_{1}^{0}$ (PA). So, by Theorem 20, we find that $U$ is $\Sigma_{1}^{0}$-sound.

The contrast between Theorems 19 and 21, illustrates well how sensitive the choice of the base theory may be already in the case where we consider $\Sigma_{1}^{0}$-axiomatizations. Moreover, this contrast may be a warning against uncritical use of Craig's trick of the form we may always replace a $\Sigma_{1}^{0}$-axiomatization by a $\Delta_{0}(\mathrm{exp})$-axiomatization. This is only unproblematic when we have a $\Sigma_{1}^{0}$-sound base theory and consider extensions of $\mathrm{EA}+\mathrm{B} \Sigma_{1}^{0}$. In all other cases, some care is needed.

Open Access This article is licensed under a Creative Commons Attribution 4.0 International License, which permits use, sharing, adaptation, distribution and reproduction in any medium or format, as long as you give appropriate credit to the original author(s) and the source, provide a link to the Creative Commons licence, and indicate if changes were made. The images or other third party material in this article are included in the article's Creative Commons licence, unless indicated otherwise in a credit line to the material. If material is not included in the article's Creative Commons licence and your intended use is not permitted by statutory regulation or exceeds the permitted use, you will need to obtain permission directly from the copyright holder. To view a copy of this licence, visit http://creativecommons.org/licenses/by/4.0/.

\section{A Examples}

For convenience, we repeat our overview of the examples.

Example $4+++$ : We take $U_{0}:=U:=$ EA and $P:=\operatorname{prov}_{\beta}$. Clearly, this $P$ satisfies all three conditions for EA. We note that our example satisfies $\mathrm{HB}$ too.

Example $5++-$ : By Theorem 16 , there is a $\Sigma_{1}^{0}$-predicate $P$ for $U:=$ EA, that satisfies Löb Conditions and the absorption principle in combination with $\square_{\beta}$. By Example 3, the predicate $P$ cannot be Fefermanian.

Here is a second example. We consider $\operatorname{cfprov}_{\beta}(x)$, which stands for cut-free provability in EA. Let's write $\square_{\beta}^{\text {cf }} A$ for $\operatorname{cfprov}(\ulcorner A\urcorner)$. We have Löb's Logic for $\square \square_{\beta}^{\text {cf }}$. See [29] and [15]. Also we easily see that $\square_{\beta}^{\text {cf }}$ satisfies the Kreisel condition. However, $\square_{\beta}^{\text {cf }}$ cannot be Fefermanian for EA over EA. If it were Fefermanian, we would have 


\begin{tabular}{lllllll}
\hline & Base & Lead & $P$ & Löb & Kreisel & Feferman \\
\hline Example 4 & EA & EA & $\Sigma_{1}^{0}$ & + & + & + \\
Example 5 & EA & EA & $\Sigma_{1}^{0}$ & + & + & - \\
Example 6 & EA & EA $+\square_{\beta} \perp$ & $\Sigma_{1}^{0}$ & + & - & + \\
& EA & EA & $\Sigma_{2}^{0}$ & & & \\
Example 7 & EA & EA & $\Sigma_{1}^{0}$ & + & - & - \\
Example 8 & PA & PA & $\Sigma_{2}^{0}$ & - & + & + \\
& EA & EA & $\Sigma_{1,1}^{0}$ & & & \\
Example 9 & EA & EA & $\Sigma_{1}^{0}$ & - & + & - \\
Example 10 & PA & PA & $\Sigma_{2}^{0}$ & - & - & + \\
& EA & EA $+\square_{\beta} \square_{\pi} \perp$ & $\Sigma_{1,1}^{0}$ & & & \\
Example 11 & EA & EA & $\Sigma_{1}^{0}$ & - & - & - \\
\hline
\end{tabular}

EA $\vdash \square_{\beta} \perp \rightarrow \square_{\beta}^{\text {cf }} \perp$. To prove that this is impossible is outside the scope of the present article. We just give the outline of the proof, so that the reader can see the basic idea.

Suppose EA $\vdash \square_{\beta} \perp \rightarrow \square_{\beta}^{\mathrm{cf}} \perp$. It follows that EA $\vdash \diamond_{\beta}^{\mathrm{cf}} \top \rightarrow \diamond_{\beta} \top$. Then, by a meta-theorem from [37], it follows that (a) $S_{2}^{1}+\diamond_{\beta}^{\mathrm{cf}} \top \vdash \diamond_{\beta}^{J} \top$, for a definable cut $J$. We also have that (b) EA interprets $S_{2}^{1}+\diamond_{\beta}^{\mathrm{cf}} \top$. Combining (a) and (b), we find that EA interprets $S_{2}^{1}+\diamond_{\beta} T$. But this contradicts the Second Incompleteness Theorem.

We note that our examples also satisfy HB. A disadvantage is that they do not work for the global version of the Löb Conditions, where the quantifiers over sentences for L2 and L3 are inside the theory. It would be interesting to have an example for this case.

Example $6+-+$ : Here is an example of a Fefermanian predicate that does satisfy the Löb Conditions and does not satisfy the Kreisel Condition. Let $U_{0}:=\mathrm{EA}, U:=$ $\mathrm{EA}+\square_{\beta} \perp$. Let $P:=\operatorname{prov}_{\gamma}$, where $\gamma(x): \leftrightarrow \beta(x) \vee x=\left\ulcorner\square_{\beta} \perp\right\urcorner$.

We have $U \vdash \square_{\gamma} \perp$, but $U \nvdash \perp$, so the Kreisel condition fails for $P$ and $U$.

We note that our example also works for $U_{0}:=U:=\mathrm{EA}+\square_{\beta} \perp$.

We provide a second example, where the base and the lead theories are sound. By Theorem 6, the predicate that represents the axioms cannot be $\Sigma_{1}^{0}$.

We take $U_{0}:=U:=$ EA. We define: $\delta(x): \leftrightarrow \beta(x) \vee\left(\diamond_{\beta}^{\top} \wedge x=\ulcorner\perp\urcorner\right)$. We note that $\delta$ numerates $\{B\}$ in EA, where $B$ is the single axiom for EA. We find:

$$
\begin{aligned}
\mathrm{EA} \vdash \square_{\delta} \perp & \leftrightarrow\left(\square_{\beta} \perp \wedge \square_{\beta} \perp\right) \vee\left(\diamond_{\beta} \top \wedge \square_{\beta}(\perp \rightarrow \perp)\right) \\
& \leftrightarrow \top .
\end{aligned}
$$

So EA $\vdash \square_{\delta} \perp$. It follows that $\operatorname{prov}_{\delta}$ is not Kreiselian and satisfies the Feferman Conditions and the Löb Conditions. We note that $\operatorname{prov}_{\delta}$ is $\Sigma_{2}^{0}$.

Example $7+--$ : Let $U_{0}:=U:=$ EA and let $P$ be $x=x$. Clearly, $P$ satisfies the Löb conditions in EA, but $P$ is not Kreiselian. Since EA is sound and $P$ is $\Sigma_{1}^{0}$, a Fefermanian $P$ must be Kreiselian. So, $P$ is also not Fefermanian. 
Example $8-++$ : The case of Fefermanian predicates that do not satisfy the Löb Conditions is among the most interesting of our cases. The study of the possibilities for such predicates for the case of extensions of Peano Arithmetic has been taken up by Taishi Kurahashi in great depth. See $[18,19]$.

A classical example of such a predicate is Feferman Provability. We define $\pi^{\star}(y): \leftrightarrow \exists x\left(\pi_{x}(y) \wedge \diamond_{\pi_{x}} \top\right)$. Let $P:=\operatorname{prov}_{\pi^{\star}}$. This predicate was introduced by Solomon Feferman in his classical paper [3]. By the essential reflexivity of PA, one finds that $\square_{\pi^{\star}}$ is Fefermanian for PA over PA. For closely related reasons $\square^{\star}$ is Kreiselian. However, $\square^{\star}$ does not satisfy the Löb Conditions. The bimodal provability logic of $\square_{\pi}$ and $\square_{\pi^{\star}}$ has been characterized by Volodya Shavrukov in [38]. For some earlier work, see $[22,28]$. We note that $\square_{\pi^{\star}}$ is $\Sigma_{2}^{0}$.

An example of quite different flavor uses the fact that EA does not verify $\Sigma_{1}^{0}$ collection. We refer the reader to [35, Subsection 6.2]. This example provides a $\Sigma_{1}^{0}$ axiomatization $\sigma$. As a consequence $\operatorname{prov}_{\sigma}$ is $\Sigma_{1,1}^{0}$. We refer the reader to e.g. [31] for a further explanation of the relevant formula hierarchy.

Example 9 -+-: Here is an example of a $P$ that satisfies the Kreisel Condition but not the Löb Conditions and the Feferman Condition. Let $U_{0}:=U:=$ EA. Let $P(x):=$ $\left.\operatorname{frov}_{\pi}(x) \wedge x \neq\ulcorner\perp\urcorner\right)$.

We note that $\mathrm{L} 2$ fails for $P$ over EA. This shows that $P$ does not satisfy the Löb Conditions and, hence, cannot be Fefermanian.

Example $10--+$ : The examples are adaptations of the predicates and theories in Example 8. We just add something to make the examples non-Kreiselian. We use the notations of Example 8.

We give our first example of a non-Kreiselian Fefermanian predicate that does not satisfy the Löb Conditions. Let $U_{0}:=U:=$ PA. We take:

$$
\pi^{\circ}(x): \leftrightarrow \pi^{\star}(x) \vee\left(\diamond_{\pi}^{\top} \wedge x=\left\ulcorner\square_{\pi} \perp\right\urcorner\right) .
$$

Let $P:=\operatorname{prov}_{\pi^{\circ}}$.

It is easily seen that $\pi^{\circ}$ numerates the axioms of PA in PA. We have, using the fact that we have $\mathrm{HB}$ for $\square_{\pi^{\star}}$ :

$$
\begin{aligned}
\mathrm{PA} \vdash \square_{\pi^{\circ}} \square_{\pi} \perp & \leftrightarrow\left(\square_{\pi} \perp \wedge \square_{\pi^{\star}} \square_{\pi} \perp\right) \vee\left(\diamond_{\pi} \top \wedge \square_{\pi^{\star}}\left(\square_{\pi} \perp \rightarrow \square_{\pi} \perp\right)\right) \\
& \leftrightarrow \square_{\pi} \perp \vee \diamond_{\pi} \top \\
& \leftrightarrow \top
\end{aligned}
$$

It follows that PA $\vdash \square_{\pi} \circ \square_{\pi} \perp$. However, PA $\nvdash \square_{\pi} \perp$, so prov o $^{\circ}$ is not Kreiselian. We have:

$$
\begin{aligned}
\mathrm{PA} \vdash \square_{\pi^{\circ}} \perp & \leftrightarrow\left(\square_{\pi} \perp \wedge \square_{\pi^{\star}} \perp\right) \vee\left(\diamond_{\pi} \top \wedge \square_{\pi^{\star}} \neg \square_{\pi} \perp\right) \\
& \leftrightarrow\left(\square_{\pi} \perp \wedge \perp\right) \vee\left(\diamond_{\pi} \top \wedge \square_{\pi} \neg \square_{\pi} \perp\right) \\
& \leftrightarrow \diamond_{\pi} \top \wedge \square_{\pi} \perp \\
& \leftrightarrow \perp
\end{aligned}
$$


So PA $\vdash \neg \square_{\pi^{\circ}} \perp$. Thus, $\square_{\pi^{\circ}}$ cannot satisfy the Löb Conditions. Finally, $\square$ ' is clearly $\Sigma_{2}^{0}$.

Here is our second example. The presentation of our example presupposes that the reader has [35, Subsection 6.2] at hand. The predicate $\sigma$ is imported here from that paper. We take $U_{0}:=\mathrm{EA}, U:=\mathrm{EA}+\square_{\beta} \square_{\pi} \perp$. We define

$$
\sigma^{\circ}(x): \leftrightarrow \sigma(x) \vee x=\left\ulcorner\square_{\beta} \square_{\pi} \perp\right\urcorner .
$$

Clearly, $\sigma^{\circ}$ numerates the axioms of $U$ in EA. We take $P(x):=\operatorname{prov}_{\sigma^{\circ}}(x)$. Evidently, $P$ is Fefermanian for $\mathrm{EA}+\square_{\beta} \square_{\pi} \perp$ over EA.

Since, we have EA $\vdash \square_{\beta} C \rightarrow \square_{\sigma} C$ and EA $\vdash \square_{\sigma} C \rightarrow \square_{\sigma^{\circ}} C$. We find $U \vdash$ $\square_{\sigma} \circ \square_{\pi} \perp$. Suppose we would have $U \vdash \square_{\pi} \perp$. In would follow that EA $+\square_{\beta} \square_{\pi} \perp \vdash$ $\square_{\pi} \perp$, and, hence, EA $\vdash \square_{\pi} \perp$. Quod non. Thus $U \nvdash \square_{\pi} \perp$. So, $P$ is not Kreiselian.

We note that over $U$ we have, by $\Sigma_{1}^{0}$-completeness, that $\square_{\sigma}$ and $\square_{\sigma^{\circ}}$ coincide also in iterated $\square_{\sigma}$-contexts. Suppose $\square_{\sigma^{\circ}}$ satisfies the Löb Conditions over $U$. It follows that $\square_{\sigma}$ also satisfies the Löb Conditions over $U$. So, a fortiori, we find $U \vdash \square_{\sigma} \diamond_{\sigma} \top \rightarrow \square_{\sigma} \perp$. By Lemma 6.11 and Lemma 6.12 of [35, Subsection 6.2], we find:

$$
\mathrm{EA}+\square_{\beta} \square_{\pi} \perp \vdash\left(\mathrm{S}^{\star} \vee \square_{\beta} \perp\right) \rightarrow\left(\left(\mathrm{S}^{\star} \wedge \square_{\beta} \square_{\beta} \perp\right) \vee \square_{\beta} \perp\right)
$$

It follows that EA $+\square_{\pi} \perp+\mathrm{S}^{\star} \vdash \square_{\beta} \square_{\beta} \perp$. However, we can construct a model of $\mathrm{EA}+\square_{\pi} \perp+\mathrm{S}^{\star}+\neg \square_{\beta} \square_{\beta} \perp$ using the construction described in [35, Subsection 6.2].

Example $11---$ : We take $U_{0}:=U:=$ EA and $P(x):=\perp$. It is clear that $P$ does not satisfy the Löb Conditions. Nor is it Kreiselian or Fefermanian.

\section{References}

1. Ardeshir, M., Mojtahedi, M.: The $\Sigma_{1}$-provability logic of HA. Ann. Pure Appl. Log. 169(10), 997-1043 (2018)

2. Beklemishev, L.D.: On the classification of propositional provability logics. Math. USSR-Izv. 35(2), 247-275 (1990)

3. Feferman, S.: Arithmetization of metamathematics in a general setting. Fundam. Math. 49, 35-92 (1960)

4. Freund, A.: Slow reflection. Ann. Pure Appl. Log. 168(12), 2103-2128 (2017)

5. Freund, A., Pakhomov, F.: Short proofs for slow consistency. Notre Dame J. Formal Log. 61(1), 31-49 (2020)

6. Friedman, S.D., Rathjen, M., Weiermann, A.: Slow consistency. Ann. Pure Appl. Log. 164(3), 382-393 (2013)

7. Grabmayr, B.: On the invariance of Gödel's Second Theorem with regard to numberings. (2018). ArXiv preprint, arXiv: 1803.08392

8. Guaspari, D.: Partially conservative extensions of arithmetic. Trans. Am. Math. Soc. 254, 47-68 (1979)

9. Henk, P.: Nonstandard provability for Peano arithmetic, a modal perspective. Ph.D thesis, ILLC, Amsterdam (2016). http://www.illc.uva.nl/Research/Publications/Dissertations/DS/

10. Hájek, P., Pudlák, P.: Metamathematics of First-Order Arithmetic, Perspectives in Mathematical Logic. Springer, Berlin (1993)

11. Henk, P., Pakhomov, F.: Slow and ordinary provability for Peano arithmetic (2016). ArXiv preprint arXiv: 1602.01822 
12. Henk, P., Visser, A.: Interpretability suprema in Peano arithmetic. Arch. Math. Log. 56(5-6), 555-584 (2017)

13. Halbach, V., Visser, A.: Self-reference in arithmetic I. Rev. Symb. Log. 7, 671-691 (2014)

14. Henk, P., Yu Shavrukov, V.: A Solovay function for the least 1-inconsistent subtheory of PA. ILLC e-prints, ILLC (2016). https://eprints.illc.uva.n1/567/

15. Kalsbeek, M.B.: Towards the interpretability logic of I $\Delta_{0}+E X P$. Logic Group Preprint Series 61, Faculty of Humanities, Philosophy, Utrecht University (1991). https://lgps.sites.uu.nl

16. Kreisel, G.: On a problem of Henkin's. Indag. Math. 15, 405-406 (1953)

17. Kreisel, G.: On weak completeness of intuitionistic predicate logic. J. Symb. Log. 27(2), 139-158 (1962)

18. Kurahashi, T.: Arithmetical completeness theorem for modal logic K. Stud. Log. 106(2), 219-235 (2018)

19. Kurahashi, T.: Arithmetical soundness and completeness for $\Sigma_{2}$-numerations. Stud. Log. 106(6), 11811196 (2018)

20. Lindström, P.: Aspects of Incompleteness. Lecture Notes in Logic, vol. 10. ASL/A.K. Peters, Natick (2003)

21. Löb, M.H.: Solution of a problem of Leon Henkin. J. Symb. Log. 20, 115-118 (1955)

22. Montagna, F.: On the algebraization of a Feferman's predicate (the algebraization of theories which express Theor; X). Stud. Log. 37, 221-236 (1978)

23. Pakhomov, F.: Solovay's completeness without fixed points. In: Kennedy, J., de Queiroz, R.J.G.B. (eds.) Logic, Language, Information, and Computation, pp. 281-294. Springer, Berlin, Heidelberg (2017)

24. Parikh, R.: Existence and feasibility in arithmetic. J. Symb. Log. 36(3), 494-508 (1971)

25. Quine, W.V.: Mathematical Logic, Revised edn. Harvard University Press, Cambridge (1996)

26. Rathjen, M.: Long sequences of descending theories and other miscellanea on slow consistency. J. Log. Appl. 4(4), 1411-1426 (2017). Special issue dedicated to Gregori Mints

27. Smullyan, R.M.: Theory of Formal Systems, Annals of Mathematics Studies, vol. 47. Princeton University Press, Princeton (1961)

28. Visser, A.: Peano's smart children: a provability logical study of systems with built-in consistency. Notre Dame J. Formal Log. 30(2), 161-196 (1989)

29. Visser, A.: Interpretability logic. In: Petkov, P.P. (ed.) Mathematical Logic, Proceedings of the Heyting 1988 summer school in Varna, Bulgaria, pp. 175-209. Plenum Press, Boston (1990)

30. Visser, A.: The second incompleteness theorem and bounded interpretations. Stud. Log. 100(1-2), 399-418 (2012). https://doi.org/10.1007/s11225-012-9385-z

31. Visser, A.: Peano Corto and Peano Basso: a study of local induction in the context of weak theories. Math. Log. Q. 60(1-2), 92-117 (2014)

32. Visser, A.: Oracle bites theory. In: Gosh, S., Szymanik, J. (eds.) The Facts Matter, Essays on Logic and Cognition in Honour of Rineke Verbrugge, pp. 133-147. College Publications, London (2015)

33. Visser, A.: The second incompleteness theorem: reflections and ruminations. In: Horsten, L., Welch, P. (eds.) Gödel's Disjunction: The Scope and Limits of Mathematical Knowledge, pp. 67-90. Oxford University Press, Oxford (2016)

34. Visser, A.: Transductions in arithmetic. Ann. Pure Appl. Log. 167(3), 211-234 (2016)

35. Visser, A.: Another look at the second incompleteness theorem. Rev. Symb. Log. 13(2), 1-27 (2019)

36. Visser, A., Zoethout, J.: Provability logic and the completeness principle. Ann. Pure Appl. Log. 170(6), 718-753 (2019)

37. Wilkie, A.J., Paris, J.B.: On the scheme of induction for bounded arithmetic formulas. Ann. Pure Appl. Log. 35, 261-302 (1987)

38. Yu Shavrukov, V.: A smart child of Peano's. Notre Dame J. Formal Log. 35, 161-185 (1994)

Publisher's Note Springer Nature remains neutral with regard to jurisdictional claims in published maps and institutional affiliations. 